\title{
Statistical Model of Multicomponent-Fuel Drop Evaporation for Many-Drop Flow Simulations
}

\author{
K. G. Harstad* \\ Jet Propulsion Laboratory, California Institute of Technology, Pasadena, California 91109 \\ P. C. Le Clercq ${ }^{\dagger}$ \\ California Institute of Technology, Pasadena, California 91109 \\ and \\ J. Bellan \\ Jet Propulsion Laboratory, California Institute of Technology, Pasadena, California 91109
}

\begin{abstract}
A statistical formulation is developed describing the composition in an evaporating multicomponent-fuel liquid drop and in the gas phase surrounding it. When a complementary discrete-component model is used, it is shown that, when drops are immersed in a carrier gas containing fuel vapor, condensation of species onto the drop results in the development of a minor peak in the liquid composition probability distribution function (PDF). This peak leads to a PDF shape that can be viewed as a combination of two gamma PDFs, which is determined by five parameters. A model is developed for calculating the parameters of the two combined gamma PDFs. Extensive tests of the model for both diesel and gasoline show that the PDF results replicate accurately the discrete model predictions. Most important, the mean and variance of the composition at the drop surface are in excellent agreement with the discrete model. Results from the model show that although the second peak is minor for the liquid PDF, its corresponding peak for the vapor distribution at the drop surface has a comparable magnitude to and sometimes exceeds that corresponding to the first peak. Four-parameter models are also exercised, and it is shown that they are unable to capture the physics of the problem.
\end{abstract}

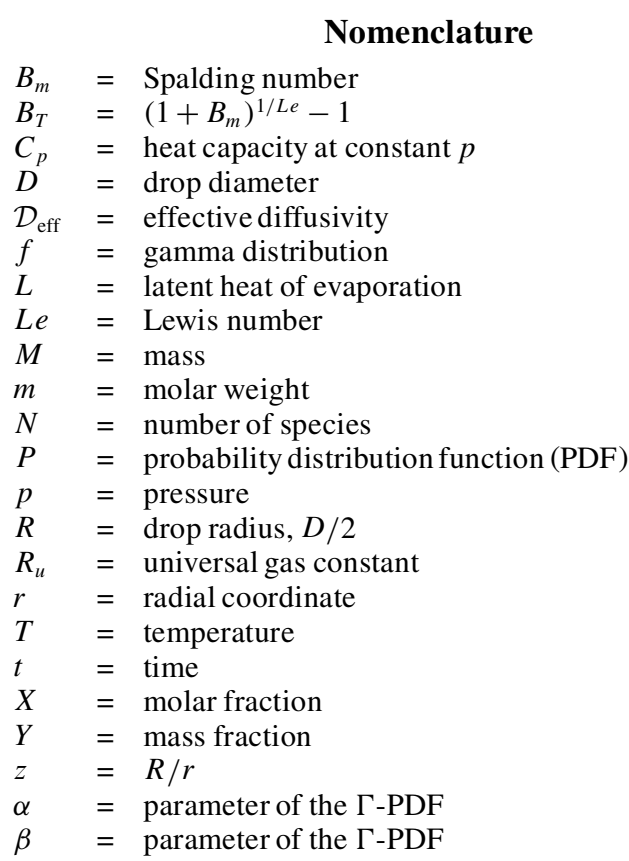

Received 14 January 2003; revision received 10 June 2003; accepted for publication 12 June 2003. Copyright (C) 2003 by the California Institute of Technology. Published by the American Institute of Aeronautics and Astronautics, Inc., with permission. Copies of this paper may be made for personal or internal use, on condition that the copier pay the $\$ 10.00$ per-copy fee to the Copyright Clearance Center, Inc., 222 Rosewood Drive, Danvers, MA 01923; include the code 0001-1452/03 \$10.00 in correspondence with the CCC.

* Senior Engineer, Thermal and Propulsion Engineering Section, Mail Stop 125-109, 4800 Oak Grove Drive.

${ }^{\dagger}$ Postdoctoral Fellow, Aeronautics Department, Mail Stop 125-109, 4800 Oak Grove Drive. Member AIAA.

${ }^{\ddagger}$ Senior Research Scientist, Thermal and Propulsion Engineering Section, Mail Stop 125-109, 4800 Oak Grove Drive. Associate Fellow AIAA.

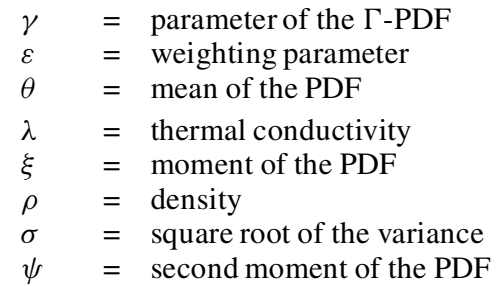

\section{Subscripts}

$a=$ ambient

$b=$ boiling point

$c=$ critical point

$d=$ drop

$g \quad=$ gas

hvy $=$ heavy species

$i=\operatorname{species} i$

$l \quad=$ liquid

$n=n$ th-order moment

$v \quad=$ vapor

$0 \quad=$ initial condition

Superscripts

$(s) \quad=$ drop surface conditions

$(\infty)=$ far-field conditions

\section{Introduction}

$\mathbf{I}$ $\mathrm{N}$ most combustion applications using liquid fuels, atomization is the method of choice in introducing the fuel in the combustion chamber. Through atomization, the liquid fuel is broken into a multitude of drops, thereby increasing the available liquid area and facilitating evaporation. Therefore, notwithstanding drop interaction effects in sprays, a liquid fuel drop represents the simplest entity that is relevant to studying fuel sprays.

Most experiments of single real-fueldrops suffer from difficulties in measuring the fuel composition from the initial condition up to the drop disappearance. The problem is that current measurement 
techniques cannot capture the drop composition during the initial heating transients even for drops composed of a binary species mixture (for example, Refs. 1-3) and so it is only the $D^{2}$ history that is documented after the initial transient (for example, Refs. 2 and 4-8). Because differential evaporation of the species occurs during the heat up time, this means that the presented $D^{2}(t)$ documents a drop whose initial composition is unknown. Sometimes, drop temperature measurements are available either at the surface, ${ }^{9}$ or more seldom inside the drop. ${ }^{10}$ In the following, we will show that neither $D^{2}$ nor the average drop temperature are reliable indicators of its chemical composition.

Detailed models of multicomponent- (MC-) fuel drops were developed in the past by Law et al. ${ }^{11}$ Lara-Urbaneja and Sirignano, ${ }^{12}$ Tong and Sirignano, ${ }^{13,14}$ and Megaridis and Sirignano, ${ }^{15}$ among others. These were, however, not intended for use in spray simulations because the computation time was prohibitive even for a single drop composed of three species. Even with current computational capabilities, the calculation time remains daunting if many millions of drops must be considered (that is, thousands of CPU hours), as in practical sprays.

Recognizing the complexity of MC fuels, but aiming at a systematic and controlled study of fuel properties influencing gas turbine combustion, Wood et al. ${ }^{16}$ and Schultz ${ }^{17}$ have developed surrogate fuels. These fuels are composed of specified 10-15 components, and the global boiling, flashing and freezing points, the saturation pressure curve, the density, the viscosity, and thermal conductivity of the original fuel are well matched to the fuels mimicked. Discrete modeling of these surrogate fuels in spray applications remains a daunting task.

This study is devoted to the modeling of MC-fuel drops containing a very large number of species. Such a model was proposed by Tamim and Hallett ${ }^{18}$ and Hallett ${ }^{19}$ and utilized by Lippert ${ }^{20}$ and Lippert and Reitz. ${ }^{21}$ That model was based on a statistical representation of the fuel composition using continuous thermodynamics (CT). CT is a theory ${ }^{22-26}$ in which the composition of a mixture is described by a probability distribution function (PDF) rather than by a series of discrete values of the concentration. Generally, this PDF is a function of all thermophysical properties of the chemical species; however, in practical applications, it can be chosen to depend on one or several properties of interest of the mixture, ${ }^{25}$ such as the relative volatility, ${ }^{22}$ the normal boiling point, the number of carbon atoms per molecule, or most conveniently for many applications, the molar weight. ${ }^{26}$ The simplification that the PDF depends only on the molar weight is possible for mixtures composed of homologous species, ${ }^{26,27}$ and such distributions, based on the gamma PDF ( $\Gamma-P D F)$, are available for diesel fuel, gasoline, and kerosene. ${ }^{18,26}$ Thus, the advantage of CT theory is that the mixture composition can be represented by a small number of parameters rather than by the prohibitively large number of parameters that would be necessary even for a discretely described surrogate fuel. The theory is based on the appropriate representation of the chemical potential for a mixture containing numerous components and uses molecular thermodynamic methods to represent the Gibbs function in terms of this PDF. The concepts are fundamental and independent of the physicochemical model chosen to represent the chemical potential. For a specified initial PDF, the evolution of the mixture is governed by thermodynamic relationships and/or conservation equations. The CT method has been successfully used for 1) calculating vapor-liquid equilibrium, ${ }^{18,28} 2$ ) computing liquidliquid equilibrium, ${ }^{28} 3$ ) simulating polymer solutions, ${ }^{26,28,29}$ 4) computing distillation, ${ }^{28}$ 5) calculating flash points ${ }^{26,29,30}$ 6) characterizing carbon plus fractions, ${ }^{29}$ and 7) modeling MC-fuel drop evaporation. . $^{181}$

However, limitations of the drop model based on the $\Gamma$-PDF ${ }^{18,19}$ were reported by Lippert. ${ }^{20}$ Specifically, unphysical results were obtained for drops evaporating in gas containing fuel vapor, or for large evaporation rates. The goal of this investigation is to formulate a more robust statistical model. Because measurements are not available for model evaluation, results from a discrete species model here provide the exact distribution that must be replicated by the model. The proposed model is evaluated by testing it un- der a wide range of conditions. To ensure that the proposed model represents the simplest formulation replicating the results of the discrete model, models of lesser complexity are formulated and exercised, and their predictions are compared to those of the discrete and proposed model. Composition distributions used in the calculations are shown in Fig. 1. The model assessment is summarized in the concluding remarks.

\section{Models of Multicomponent Drop Evaporation}

In any model, physical accuracy must be balanced against complexity. For a drop model that is usable in configurations where there are millions of drops, the goal is to capture the crucial features of the MC-fuel with a formulation that is simple enough to be one of the building blocks of a larger model. Therefore, several assumptions are made: the drop is spherical, $\rho_{l}$ is constant, liquid evaporation occurs under thermodynamic equilibrium, the carrier gas surrounding the drop obeys the perfect gas equation of state, and the gas is quasi-steady with respect to the liquid, ${ }^{31,32}$ which is justified by its much smaller characteristic time compared to that of the liquid. Furthermore, we are only interested in the average volumetric properties of the drop represented by $T_{d}$ and $Y_{i l}=M_{i} / M_{d}$, where $M_{d}=4 \pi R^{3} \rho_{l} / 3$ and

$$
\sum_{i=1}^{N} M_{i}=M_{d}
$$

The interest in average drop properties precludes consideration of differential species diffusivities and, therefore, of any phenomena resulting from such processes. The study is performed at atmospheric pressure, where solubility of the carrier gas into the liquid is negligible. The far-field conditions are assumed quiescent.

\section{A. Discrete-Species Drop Mode}

A discreterepresentationof the mixture composition as a function of the molar weight is shown in Fig. 1a, where bins in the molar weight space represent species or pseudospecies.

The traditional equations for the drop and its surrounding gas field are ${ }^{19,31}$

$$
\begin{aligned}
\frac{\mathrm{d} D^{2}}{\mathrm{~d} t} & =-\frac{8 \rho_{g}^{(s)} \mathcal{D}_{\text {eff }}}{\rho_{l}} \ln \left(1+B_{m}\right) \\
\frac{\mathrm{d} T_{d}}{\mathrm{~d} t} & =\frac{3 \lambda_{g}^{(s)}}{\rho_{l} C_{l} R^{2}}\left[\left(T^{(\infty)}-T_{d}\right) \frac{\ln \left(1+B_{T}\right)}{B_{T}}\right. \\
& \left.-\frac{L_{v}^{(s)}\left(1+B_{m}\right)-L_{v}^{(\infty)}}{C_{p}^{*} L e} \frac{\ln \left(1+B_{m}\right)}{B_{m}}\right] \\
\Omega_{g} & =\Omega_{g}^{(s)}+\frac{\left(\Omega_{g}^{(\infty)}-\Omega_{g}^{(s)}\right)\left[\left(1+B_{\Omega}\right)^{1-z}-1\right]}{B_{\Omega}}
\end{aligned}
$$

where $\Omega_{g}$ is $T_{g}$ or $Y_{i g}, B_{\Omega}$ is $B_{T}$ or $B_{m}=\left(Y_{v}^{(s)}-Y_{v}^{(\infty)}\right) /\left(1-Y_{v}^{(s)}\right)$, respectively, and $L e \equiv \lambda_{g}^{(s)} /\left(C_{p}^{*} \rho_{g}^{(s)} \mathcal{D}_{\text {eff }}\right)$. Here, $\lambda_{g}^{v(s)}$ is calculated as a function of $T_{g}^{(s)}$ using mixing rules (Appendix A) and $C_{p}^{*}=$ $Y_{v}^{(s)} C_{p v}^{(s)}+\left(1-Y_{v}^{(s)}\right)^{g}\left[C_{p a g}+\left(C_{p v}^{(s)}-C_{p a g}\right) B_{m} / \ln \left(1+B_{m}\right)\right]$. To solve the discrete problem, one needs to find $D, T_{d}$, and $Y_{i l}$; therefore, there are $N+2$ variables.

For a large number of discrete species, one may define the following statistics:

$$
\xi_{n l} \equiv \sum_{j=1}^{N}\left(m_{j}^{n} X_{j l}\right), \quad \xi_{n v} \equiv \frac{1}{X_{v}} \sum_{j=1}^{N}\left(m_{j}^{n} X_{j v}\right)
$$

where $X_{v} \equiv 1-X_{a g}$. Then, $\theta_{l}=\xi_{1 l}=m_{l}$ and $\theta_{v}=\xi_{1 v}=m_{v}$. These statistics enable the comparison between results from the statistical models based on a PDF and the discrete model. 


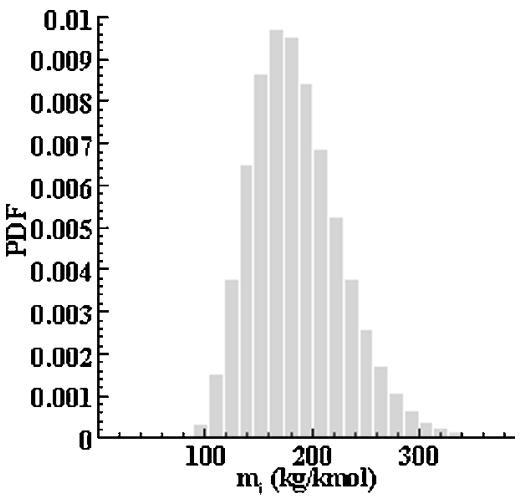

a)

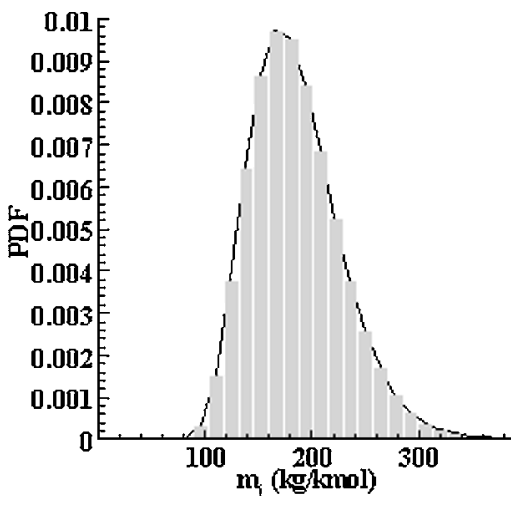

b)

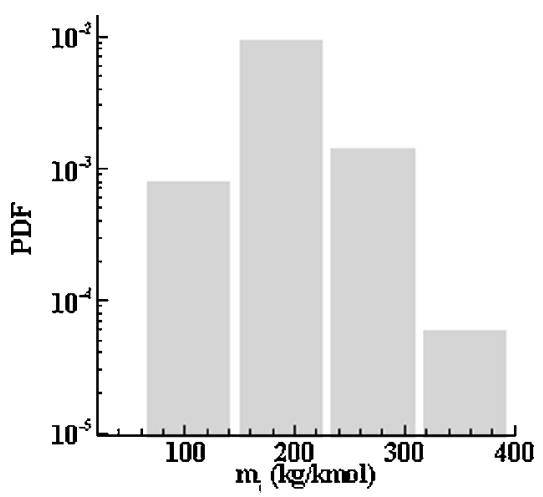

c)

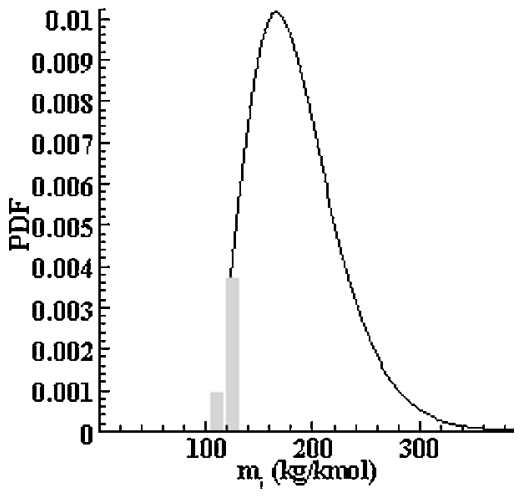

d)

Fig. 1 PDFs used in the computations: a) discrete pseudocomponents for diesel fuel, $\gamma=86 \mathrm{~kg} / \mathrm{kmol}, \theta_{l, 0}=185 \mathrm{~kg} / \mathrm{kmol}$, and $\left.\sigma_{l, 0}=43, \mathrm{~b}\right)$ single- $\Gamma$-PDF envelope of the initial discrete PDF of diesel fuel, c) PDF of diesel with four pseudocomponents, and d) PDF of diesel with two discrete light components close to the origin and the remaining modeled as a single- $\Gamma$-PDF.

These definitions combined with the liquid species conservation equation also lead to a differential equation for each moment:

$$
\begin{aligned}
\frac{\mathrm{d} \xi_{n l}}{\mathrm{~d} t} & =\frac{3 \rho_{g}^{(s)} \mathcal{D}_{\text {eff }} m_{l}}{\rho_{l} R^{2} m_{g}^{(s)}} \frac{1+B_{m}}{B_{m}} \ln \left(1+B_{m}\right)\left[\left(X_{v}^{(s)}-X_{v}^{(\infty)} \frac{1-X_{v}^{(s)}}{1-X_{v}^{(\infty)}}\right) \xi_{n l}\right. \\
& \left.-\left(X_{v}^{(s)} \xi_{n v}^{(s)}-X_{v}^{(\infty)} \frac{1-X_{v}^{(s)}}{1-X_{v}^{(\infty)}} \xi_{n v}^{(\infty)}\right)\right]
\end{aligned}
$$

These equations serve as the basis for generalizationto the statistical representation presented in our new model.

\section{B. CT Using a Single- $\Gamma$-PDF}

The representation of a MC-fuel mixture composition by the single- $\Gamma$-PDF is shown in Fig. $1 b$.

\section{Description of a Mixture Using CT Theory}

In CT form, the mole fraction of a discrete species $i$ in a hydrocarbon mixture is defined by the value of a continuous distribution function $f$ in the vicinity of the molar mass point corresponding to that species through $X_{i}=f\left(m_{i}\right) \Delta m_{i}$. Thus, for a hydrocarbon liquid fuel,

$$
X_{i l}=f_{l}\left(m_{i}\right) \Delta m_{i}
$$

For a mixture containing hydrocarbons and nonhydrocarbon species, such distribution functions describing all components are not necessarily available. An example of such a situation is that of gasoline vapor in air. To utilize the CT formulation in this situation, we note that $X_{a g}+X_{v}=1$, and use the CT formulation for the hydrocarbon as

$$
X_{i v}=X_{v} f_{v}\left(m_{i}\right) \Delta m_{i}
$$

Then, from the discrete form

$$
m_{g}=m_{a g} X_{a g}+\sum_{i=1}^{N} m_{i} X_{i v}
$$

one derives the continuous form $m_{g}=m_{a g}\left(1-X_{v}\right)+\theta_{v} X_{v}$ where

$$
\theta_{v}=\int_{0}^{\infty} f_{v}(m) m \mathrm{~d} m, \quad \psi_{v}=\int_{0}^{\infty} f_{v}(m) m^{2} \mathrm{~d} m
$$

Whitson ${ }^{29}$ used the $\Gamma$-PDF

$$
f_{\Gamma}(m)=\frac{(m-\gamma)^{\alpha-1}}{\beta^{\alpha} \Gamma(\alpha)} \exp \left[-\left(\frac{m-\gamma}{\beta}\right)\right]
$$

to characterize the high molar-weight portion of crude oils, where $\Gamma(\alpha)$ is the gamma function. The origin of $f$ is specified by $\gamma$, and its shape is determined by $\alpha$ and $\beta$. These parameters are related to $\theta_{v}, \sigma_{v}^{2}$ and $\psi_{v}$ of $f$ by $\theta_{v}=\alpha \beta+\gamma, \sigma_{v}^{2}=\alpha \beta^{2}$, and $\psi_{v}=\theta_{v}^{2}+\sigma_{v}^{2}$.

\section{Equations for a Single- $\Gamma-P D F$}

From the liquid species conservation equation one can derive an equation for $X_{i l}$. This equation is the same as Eq. (5) with $\xi_{n l}$ replaced by $X_{i l}$ and $X_{v} \xi_{n v}$ replaced by $X_{i v}$. Substituting the discrete mole fractions by their continuous forms [Eqs. (6) and (7)] in the vapor and the liquid phases, one obtains from Eq. (5) differential equationsfor $\theta_{l}$ and $\psi_{l}$ by replacing $\xi_{1 l}$ by $\theta_{l}$ and $\xi_{2 l}$ by $\psi_{l}$. Using the CT formulation in conjunction with the conservationequationsleads to relationshipsbetween the vapor molar fraction at the drop surface, $X_{v}^{(s)}$, and the distribution parameters in the liquid and between the distribution parameters in the liquid and vapor

$$
X_{v}^{(s)}=\frac{p_{\text {atm }}}{p^{(\infty)}} \frac{\exp \left\{\left[\Delta s_{f g} /\left(R_{u} T_{g}^{(s)}\right)\right]\left(T_{g}^{(s)}-A_{b}-\gamma B_{b}\right)\right\}}{\left\{1+\left[\Delta s_{f g} /\left(R_{u} T_{g}^{(s)}\right)\right] B_{b} \beta_{l}\right\}^{\alpha_{l}}}
$$




$$
\begin{gathered}
\theta_{v}^{(s)}-\gamma=\frac{\theta_{l}-\gamma}{1+\left[\Delta s_{f g} /\left(R_{u} T_{g}^{(s)}\right)\right] B_{b} \beta_{l}} \\
\left(\sigma_{v}^{(s)}\right)^{2}=\sigma_{l}^{2}\left[\frac{\theta_{v}^{(s)}-\gamma}{\theta_{l}-\gamma}\right]^{2}
\end{gathered}
$$

having assumed that $\gamma_{l}=\gamma_{v}^{(s)}=\gamma$. We also postulate that $T_{g}^{(s)}=T_{d}$. In Eqs. (10) and (11), $p_{\mathrm{atm}}=1 \mathrm{~atm}$, and the entropy of evaporation expressed using Trouton's law is $\Delta s_{f g}=m L_{v} / T_{b} \simeq 10.6 R_{u}$. Constants $A_{b}$ and $B_{b}$ are listed in Appendix A and fit $T_{b}(m)=A_{b}+B_{b} m$.

To solve the problem, one needs to calculate $D, T_{d}, \theta_{l}$, and $\psi_{l}$. Therefore, there are four variables, representing a reduction from the $N+2$ discrete-model variables; for $N>2$, it is computationally preferable to use the single- $\Gamma$-PDF at comparableaccuracy. Once the composition of the mixture is known at the drop surface, Eqs. (3) provide the composition of the gaseous mixture surrounding the drop.

\section{CT Using a Double- $\Gamma$-PDF}

The limitations of the single- $\Gamma-\mathrm{PDF}$ documented in our results comparing its predictions with those of the discrete model displayed in statistical form (discussed later), prompted our investigation into the description of the fuel composition using a combination of two $\Gamma$-PDFs,

$$
P_{l}\left(m ; \alpha_{1}, \beta_{1}, \alpha_{2}, \beta_{2}, \epsilon\right)=(1-\epsilon) f_{\Gamma}^{(1)}(m)+\epsilon f_{\Gamma}^{(2)}(m)
$$

where $f_{\Gamma}^{(q)}(m)=f_{\Gamma}\left(m ; \alpha_{q}, \beta_{q}\right)$ with $q \in[1,2], \epsilon$ is a weighting parameter, $0 \leq \epsilon \leq 1$, and

$$
\int_{\gamma}^{\infty} P_{l}(m) \mathrm{d} m=1
$$

The problem of determining $P_{l}$ can be stated as follows: Given an initial single- $\Gamma$-PDF characterized by $\theta_{0}, \beta_{0}, \gamma$, and $\epsilon=0$, is it possible to determine $P_{l}$ as a function of time? To do so, one needs to solve for the vector $\boldsymbol{\eta} \equiv\left(\alpha_{1}, \beta_{1}, \alpha_{2}, \beta_{2}, \epsilon\right)$ at each time step. If $\boldsymbol{\eta}$ is known, for $n \geq 1$

$$
\xi_{n l} \equiv \int_{\gamma}^{\infty} m^{n} P_{l}(m) \mathrm{d} m
$$

may be calculated for any value of $n$. Conversely, an inverse mapping may be defined in that given $\xi_{n l}$ for $n \in[1,5], \boldsymbol{\eta}$ may be calculated. This inverse mapping is the driving idea behind the determination of $\boldsymbol{\eta}$. The differential equations solved for $\xi_{n l}$ are the continuous form of Eq. (5), where the equation is the same except that $m_{g}^{(s)}=X_{v}^{(s)} \theta_{v}^{(s)}+\left(1-X_{v}^{(s)}\right) m_{a g}$ and $m_{l}$ is replaced by $\theta_{l}$. In statistical form, $\xi_{n v}^{(\infty)}$ is specified, and by definition

$$
\xi_{n v}^{(s)}=\int m^{n} P_{v}^{(s)}(m) \mathrm{d} m
$$

Raoult's law in continuous form is used to relate $P_{v}^{(s)}$, to $P_{l}^{(s)}$ and $X_{v}^{(s)}$ through

$$
P_{v}^{(s)}=\left[p_{\mathrm{atm}} / X_{v}^{(s)} p^{(\infty)}\right] \exp \left\{\left(m L_{v} / R_{u} T_{b}\right)\left[1-T_{b}(m) / T_{d}\right]\right\} P_{l}^{(s)}
$$

Similar to the situation for the single- $\Gamma$-PDF, once the composition of the mixture is known at the drop surface, Eqs. (3) provide the composition of the gaseous mixture surrounding the drop.

The form of $\xi_{n l}$ for $n \in[1,5]$ is calculated as a function of $\boldsymbol{\eta}$ and is presented in Appendix B. By definition, $\xi_{1 l} \equiv \theta, \xi_{2 l} \equiv \theta^{2}+\sigma^{2}$, and $\beta=\sigma^{2} /(\theta-\gamma)$. Each $\xi_{n l}$ can be written as

$$
\xi_{n l}=(1-\epsilon) \xi_{n l}^{(1)}+\epsilon \xi_{n l}^{(2)}
$$

where $\xi_{1 l}^{(j)}=\theta_{j}$ and $\xi_{2 l}^{(j)}=\theta_{j}^{2}+\sigma_{j}^{2}$, thus correspondingto the single$\Gamma$-PDF form. Because the double- $\Gamma$-PDF can be considered as a departure from the single- $\Gamma-\mathrm{PDF}$, it is natural to introduce the concept of excess moments by defining $\xi_{n l}^{*}$ as being the moments of a single- $\Gamma$-PDF that would have the same $\xi_{1 l}$ and $\xi_{2 l}$ values as a given $P_{l}$. Thus,

$$
\xi_{n}^{\prime} \equiv \xi_{n l}-\xi_{n l}^{*}
$$

can be considered to be the departure, or excess, from that form. By definition, $\xi_{1}^{\prime}=\xi_{2}^{\prime}=0$, and a double- $\Gamma$-PDF then corresponds to $\xi_{n}^{\prime} \neq 0$ for $n \geq 3$. Because $\xi_{1 l}$ and $\xi_{2 l}$ determine $\theta$ and $\sigma$ (or $\beta$ ), the problem of determining $\boldsymbol{\eta}$ from the moments can, thus, be further reduced to the inverse map defined by $\left[\xi_{n}^{\prime} \neq 0\right.$ for $\left.n \geq 3 \rightarrow\left(\theta^{\prime}, \beta^{\prime}, \omega\right)\right]$, where $\Delta \theta \equiv \theta_{1}-\theta_{2}$ and $\theta^{\prime} \equiv(1-2 \epsilon) \Delta \theta$, $\beta^{\prime} \equiv \epsilon(1-\epsilon)(\Delta \theta)^{2} /(\theta-\gamma)$, and $\omega \equiv\left(\beta_{1}-\beta_{2}\right) / \Delta \theta$. Because $\theta_{j}>$ $\gamma$, then $\beta^{\prime} \geq 0$. For definiteness, we choose the PDF indexed by 1 to be located at a larger mean and be in magnitude larger than that indexed by 2 , so that $\Delta \theta>0, \epsilon \leq 0.5$, and $\theta^{\prime} \geq 0$. An additionalconstraint imposed by physics is that $\beta_{j}>0$. The exact form of $\xi_{n}^{\prime}$ for $n \in[3,5]$ is calculable, and the results show that $\xi_{5}^{\prime}$ is particularly complex, making the finding of a reverse mapping based on all $\xi_{n}^{\prime}$ for $n \in[3,5]$ a formidable task. A further problem in reaching a quantitative agreement with the statistics from the discrete model is that those statistics may not be entirely of the form of Eq. (12), in which case the $\xi_{n}^{\prime}$ values are only an approximation to the true departure from the single $\Gamma$-PDF. For this reason, a simplification is introduced by restricting the inverse mapping to $\left[\xi_{n}^{\prime}\right.$ for $\left.n=[3,4] \rightarrow\left(\theta^{\prime}, \beta^{\prime}\right)\right]$ and $\omega$ is further considered an empirical parameter; expressions for $\xi_{3}^{\prime}$ and $\xi_{4}^{\prime}$ are provided in Appendix B. Therefore, the employed inverse mapping is only approximate, with possible consequences on the accuracy of the model. That is, the model is expected to be in some cases only qualitative when compared to the statistics from the discrete model solution.

Cumbersome and tedious calculations show that the solution to the inverse mapping is obtained by solving a cubic algebraic equation for one of the variables and that the other is found from a simple linear algebraic relationship (Appendix B). In choosing the root of the cubic equation, the criterion is that $\beta^{\prime}$ should be the smallest positive root because it is the one yielding the smallest $\epsilon$, representing the smallest departure from the single- $\Gamma-P D F$. For specific values of $\omega$, the cubic equation has easily computed roots. A thorough study of the root and solution behavior for various values of $\omega$ allowed a classification of the solution in two types according to the sign of $\Lambda \equiv\left[-2 \xi_{3}^{\prime} /(\theta-\gamma)-(\beta+\theta-\gamma)\left(7 \beta+3 \theta+\gamma-\xi_{4}^{\prime} / \xi_{3}^{\prime}\right)\right] /$ $(\beta+\theta-\gamma)^{2}$. Specifically, condition $\Lambda<0$ corresponds to larger $\beta_{2},-2 \leq \omega=-0.62+k_{f} \Lambda \leq-0.62$, and $k_{f}>0$, and condition $\Lambda>0$ corresponds to smaller $\beta_{2},-0.38 \leq \omega=-0.38+k_{s} \Lambda \leq$ $w[\beta /(\theta-\gamma)]$ and $k_{s}>0$ and $0 \leq w<1$. Optimal values used in the calculations presented in the Results section are $k_{f}=0.4, k_{s}=0.8$, and $w=0.67$. The condition $\Lambda \simeq 0$ is avoided because it may lead to an irregularbehavior. Once $\theta^{\prime}$ and $\beta^{\prime}$ are found, the other parameters of $P_{l}$ are calculated as follows:

$$
\begin{gathered}
\Delta \theta=\sqrt{\left(\theta^{\prime}\right)^{2}+4(\theta-\gamma) \beta^{\prime}}, \quad \epsilon=0.5\left(1-\theta^{\prime} / \Delta \theta\right) \\
\theta_{1}=\theta+\epsilon \Delta \theta, \quad \theta_{2}=\theta-(1-\epsilon) \Delta \theta \\
\beta_{1}=\beta-(1+\omega) \beta^{\prime}+\omega \epsilon \Delta \theta \\
\beta_{2}=\beta-(1+\omega) \beta^{\prime}-\omega(1-\epsilon) \Delta \theta
\end{gathered}
$$

There are six variablesin the problem: $D, T_{d}$, and $\xi_{i l}$ for $i \in[1,4]$. Because in the discrete problem there are $N+2$ variables, there is an advantage in adopting the double- $\Gamma$-PDF formulations if $N>4$.

\section{Simplified Models}

The complexity of the double- $\Gamma$-PDF approach naturally leads to inquiring if a reasonable approximation of the discrete model results cannot be accomplished with a lesser number of parameters. Equation (12) shows that the double- $\Gamma$-PDF has five parameters. Thus, we evaluate here models that require the computation of only four parameters.

\section{Four Pseudocomponents Model}

Each pseudocomponent is defined by its molar weight, which is an average over several discrete component molar weights. The initial distribution of discrete components is divided into four bins (Fig. 1c); this is the simplest four-parameter approach. With 32 
discrete components and 4 pseudocomponents, there are 8 species per bin, whose molar weight is defined as

$$
m_{p i}=\frac{1}{8} \sum_{k=K}^{K+7} m_{k}
$$

$K=8 i-7$ and $i \in[1,4]$. The four liquid mole fractions are then given by integrating the initial $\Gamma$ distribution of discrete components over the corresponding eight species

$$
X_{p i}=\int_{m_{K}-\Delta m / 2}^{m_{K+7}-\Delta m / 2} f_{l}(m) \mathrm{d} m
$$

and the resulting mass fractions are $Y_{p i}=m_{p i} X_{p i} / m_{l}$. The liquid mass fractions timewise evolution is that of the discrete model, the unknown being $Y_{p i}$ instead of $Y_{i l}$. Basically, the conservation equations are those of the discrete model; however, instead of solving $N$ equations for the species in the mixture, here only four equations must be solved for the pseudospecies. Therefore, there are six variables in the problem: four mass fractions in the liquid phase, $D$, and $T_{d}$.

\section{Mixed Models}

In the original CT theory, Aris and Gavalas ${ }^{24}$ used generalized functions to describe any type of mixture containing many components. That is, a mixture may be idealized as a superposition of species belonging to a continuous distribution and of discrete species. The generalized molar weight distribution function is thus written as

$$
P_{l}(m)=f(m)+\sum_{i=1}^{N} X_{l i} \delta\left(m-m_{i}\right)
$$

This description is here called a mixed model, being a combination of discrete and continuous models. Because the goal is to have no more than four equations for describing the evolution of the fuel composition, and given that the continuous part of the model based on the single- $\Gamma$-PDF already requires two equations, this leaves room for only two discrete components.

The first mixed model, shown in Fig. 1d, uses the lighter components of the initial distribution as discrete components and the remaining is described by the single- $\Gamma$-PDF. To understand the formulation, consider the liquid composition. By definition, the sum of all mole fractions is unity:

$$
\sum_{i=1}^{2} X_{l, i}+X_{l, \mathrm{hvy}} \int_{\gamma_{3}} f_{l}(m) \mathrm{d} m=1
$$

where $\gamma_{3}=m_{2}+7$,

$$
\int_{\gamma_{3}} f_{l}(m) \mathrm{d} m=1
$$

$X_{l, 1}$ and $X_{l, 2}$ are the two discrete mole fractions and $X_{l, \text { hvy }}$ is the remaining mole fraction of heavier components defined by

$$
X_{l, \mathrm{hvy}}=1-\sum_{i=1}^{2} X_{l, i}
$$

By definition,

$$
\begin{gathered}
\xi_{n l}=\sum_{i=1}^{2} X_{l, i} m_{i}^{n}+X_{l, \mathrm{hvy}} \int_{\gamma_{3}} f_{l}(m) m^{n} \mathrm{~d} m \\
\xi_{1 l}=\theta_{l}, \quad \xi_{2 l}=\psi_{l}
\end{gathered}
$$

In the far-field vapor phase, the remaining mole fraction of heavier components is

$$
X_{v, \text { hvy }}^{(\infty)}=X_{v}^{(\infty)}-\sum_{i=1}^{2} X_{v, i}^{(\infty)}
$$

where

$$
\begin{gathered}
X_{v, i}^{(\infty)}=X_{v}^{(\infty)} \frac{\left(m_{i}-\gamma\right)^{\alpha_{v}-1}}{\beta_{v}^{\alpha_{v}} \Gamma\left(\alpha_{v}\right)} \exp \left[-\frac{\left(m_{i}-\gamma\right)}{\beta_{v}}\right] \times \Delta m \\
\xi_{n v}^{(\infty)}=\sum_{i=1}^{2} X_{v, i}^{(\infty)} m_{i}^{n}+X_{v, \text { hvy }}^{(\infty)} \int_{\gamma_{3}} f_{v}(m) m^{n} \mathrm{~d} m \\
\xi_{1 v}^{(\infty)}=\theta_{v}^{(\infty)}, \quad \xi_{2 v}^{(\infty)}=\psi_{v}^{(\infty)}
\end{gathered}
$$

Aside from $X_{l, 1}$ and $X_{l, 2}$ and $D$ and $T_{d}$, the two remaining variables for the liquid are

$$
\theta_{l, \text { hvy }}=\int_{\gamma_{3}} f_{l}(m) m \mathrm{~d} m, \quad \psi_{l, \text { hvy }}=\int_{\gamma_{3}} f_{l}(m) m^{2} \mathrm{~d} m
$$

whose time evolution is given by

$$
\begin{gathered}
\frac{\mathrm{d} \theta_{l, \text { hvy }}}{\mathrm{d} t}=\frac{1}{X_{l, \text { hvy }}}\left[\frac{\mathrm{d} \theta_{l}}{\mathrm{~d} t}-m_{1} \frac{\mathrm{d} X_{l, 1}}{\mathrm{~d} t}-m_{2} \frac{\mathrm{d} X_{l, 2}}{\mathrm{~d} t}-\theta_{l, \text { hvy }} \frac{\mathrm{d} X_{l, \text { hvy }}}{\mathrm{d} t}\right] \\
\frac{\mathrm{d} \psi_{l, \text { hvy }}}{\mathrm{d} t}=\frac{1}{X_{l, \text { hvy }}}\left[\frac{\mathrm{d} \psi_{l}}{\mathrm{~d} t}-m_{1}^{2} \frac{\mathrm{d} X_{l, 1}}{\mathrm{~d} t}-m_{2}^{2} \frac{\mathrm{d} X_{l, 2}}{\mathrm{~d} t}-\psi_{l, \text { hvy }} \frac{\mathrm{d} X_{l, \text { hvy }}}{\mathrm{d} t}\right]
\end{gathered}
$$

where the calculation of $\mathrm{d} \theta_{l} / \mathrm{d} t$ and $\mathrm{d} \psi_{l} / \mathrm{d} t$ was discussed earlier. Equations for the two discrete mole fractions are derived from the liquid discrete-species conservation equation. There are six unknowns: four composition variables in the liquid, $D$, and $T_{d}$. This mixed model is designated as M1.

To encompass a wider range of light components in the discrete part of a mixed PDF, another model, called M2, is developed that uses two pseudocomponents instead of two discrete components as in M1. An average over two or three (depending on the fuel) discrete components is used to define these two pseudocomponents; as in $\mathrm{M} 1$, the remaining part of the PDF is modeled by a single- $\Gamma-\mathrm{PDF}$. For example, when a pseudocomponent is defined from averaging over three components, its molar weight is

$$
m_{p i}=\frac{1}{3} \sum_{k=K}^{K+2} m_{k}
$$

with $K=1$ for $m_{p 1}$ and $K=4$ for $m_{p 2}$. Taking the same initial single- $\Gamma$-PDF, we approximate a pseudocomponentinitial and farfield mole fraction by

$$
\begin{array}{r}
X_{l, p i}=\frac{\left(m_{p i}-\gamma\right)^{\alpha_{l}-1}}{\beta_{l}^{\alpha_{l}} \Gamma\left(\alpha_{l}\right)} \exp \left[-\frac{\left(m_{p i}-\gamma\right)}{\beta_{l}}\right] \times 3 \Delta m \\
X_{v, p i}^{(\infty)}=X_{v}^{(\infty)} \frac{\left(m_{p i}-\gamma\right)^{\alpha_{v}-1}}{\beta_{v}^{\alpha_{v}} \Gamma\left(\alpha_{v}\right)} \exp \left[-\frac{\left(m_{p i}-\gamma\right)}{\beta_{v}}\right] \times 3 \Delta m
\end{array}
$$

Similarly to the M1 model, the mole fraction of the complementary part of the mixture that is composed of heavier components is defined by

$$
X_{l, \mathrm{hvy}}=1-\sum_{i=1}^{2} X_{l, p i}, \quad X_{v, \mathrm{hvy}}^{(\infty)}=X_{v, p i}^{(\infty)}-\sum_{i=1}^{2} X_{v, p i}^{(\infty)}
$$

The mean and the second moment of the PDF are defined similarly to the M1 model through Eqs. (21) in the liquid phase and Eqs. (22) in the gas phase. In all of these four equations, the discrete molar weights must be replaced by the pseudocomponent $m_{p 1}$ and $m_{p 2}$ values. The same strategy is used in Eqs. (23) and (24) that are solved for $\theta_{l, \text { hvy }}$ and $\psi_{l, \text { hvy }}$. There are six variables: $D, T_{d}$, two discrete components, and two parameters for the single- $\Gamma-\mathrm{PDF}$. 


\section{Results}

All models presented were exercised for the same initial and boundary conditions. The ordinary differential equations were solved using a fourth-order Fehlberg Runge-Kutta method with variable step size and the relative error tolerance was $10^{-5}$. The statistics extracted from the discrete model served as a reference, representing the desired behavior of the statistical or mixed models.

\section{A. General Considerations}

The evaporation rate and the drop temperature evolutions are strongly dependent on liquid- and gas-phase thermophysical properties. Generally, empirical or semi-empirical correlations used for the thermophysical properties are functions of $m_{i}$, or of $m_{i}$ and $T$. For each thermophysical property, the same correlation is used for all models. All correlations are presented in Appendix A.

Commercial fuels are composed of hundreds of species. Whereas in principle a discrete distribution model can reproduce a fuel with species belonging to many chemical families of hydrocarbons, for comparing results with those from CT-based models, we restrict our study to homologous families and specifically to paraffins. The lowest $m$ is specified according to the fuel, and for paraffins, the molar weight difference between adjacent species is $14 \mathrm{~kg} / \mathrm{kmol}$. This 32-species distribution in Fig.1a (only 18 of the species are
Table 1 Initial and boundary conditions

\begin{tabular}{|c|c|c|c|c|c|c|}
\hline $\operatorname{Run}^{\mathrm{a}}$ & Fuel & $X_{v}^{(\infty)}\left(Y_{v}^{(\infty)}\right)$ & $\theta_{v}^{(\infty)}, \mathrm{kg} / \mathrm{kmol}$ & $\sigma_{v}^{(\infty)}$ & Le & $T_{g}^{(\infty)}, \mathrm{K}$ \\
\hline 1 & Diesel $^{\mathrm{b}}$ & 0 & 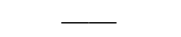 & 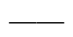 & 0.5 & 1000 \\
\hline 2 & Diesel & $0.5(0.82)$ & 131.3 & 24.4 & 0.5 & 1000 \\
\hline 3 & Diesel & $0.5(0.82)$ & 131.3 & 24.4 & 0.5 & 1200 \\
\hline 4 & Diesel & $0.5(0.82)$ & 131.3 & 24.4 & 0.5 & 600 \\
\hline 5 & Diesel & $0.3(0.66)$ & 131.3 & 24.4 & 0.5 & 1000 \\
\hline 6 & Diesel & $0.1(0.33)$ & 131.3 & 24.4 & 0.5 & 1000 \\
\hline 7 & Diesel & $0.5(0.82)$ & 120 & 18 & 0.5 & 1000 \\
\hline 8 & Diesel & $0.5(0.82)$ & 140 & 28 & 0.5 & 1000 \\
\hline 9 & Diesel & $0.5(0.82)$ & 131.3 & 24.4 & 1 & 1000 \\
\hline 10 & Diesel & $0.5(0.82)$ & 131.3 & 24.4 & 2 & 1000 \\
\hline 11 & Gasoline $^{c}$ & $0.5(0.66)$ & 58 & 14 & 0.5 & 1000 \\
\hline 12 & Gasoline & $0.5(0.66)$ & 58 & 14 & 0.5 & 1200 \\
\hline 13 & Gasoline & $0.5(0.66)$ & 58 & 14 & 0.5 & 600 \\
\hline 14 & Gasoline & $0.3(0.46)$ & 58 & 14 & 0.5 & 1000 \\
\hline 15 & Gasoline & $0.1(0.18)$ & 58 & 14 & 0.5 & 1000 \\
\hline 16 & Gasoline & $0.5(0.66)$ & 48 & 8 & 0.5 & 1000 \\
\hline 17 & Gasoline & $0.5(0.66)$ & 68 & 18 & 0.5 & 1000 \\
\hline 18 & Gasoline & $0.5(0.66)$ & 58 & 14 & 1 & 1000 \\
\hline 19 & Gasoline & $0.5(0.66)$ & 58 & 14 & 2 & 1000 \\
\hline
\end{tabular}

${ }^{\mathrm{a}} D_{0}=0.1 \mathrm{~mm}$ and $T_{d, 0}=300 \mathrm{~K}$.

${ }^{\mathrm{b}}$ For all diesel simulations $\theta_{l}=185 \mathrm{~kg} / \mathrm{kmol}, \sigma_{l}=43 \mathrm{~kg} / \mathrm{kmol}$, and $\gamma_{l}=86 \mathrm{~kg} / \mathrm{kmol}$. ${ }^{c}$ Gasoline composition is defined by $\theta_{l}=88 \mathrm{~kg} / \mathrm{kmol}, \sigma_{l}=31.5 \mathrm{~kg} / \mathrm{kmol}$ and $\gamma_{l}=30 \mathrm{~kg} / \mathrm{kmol}$.

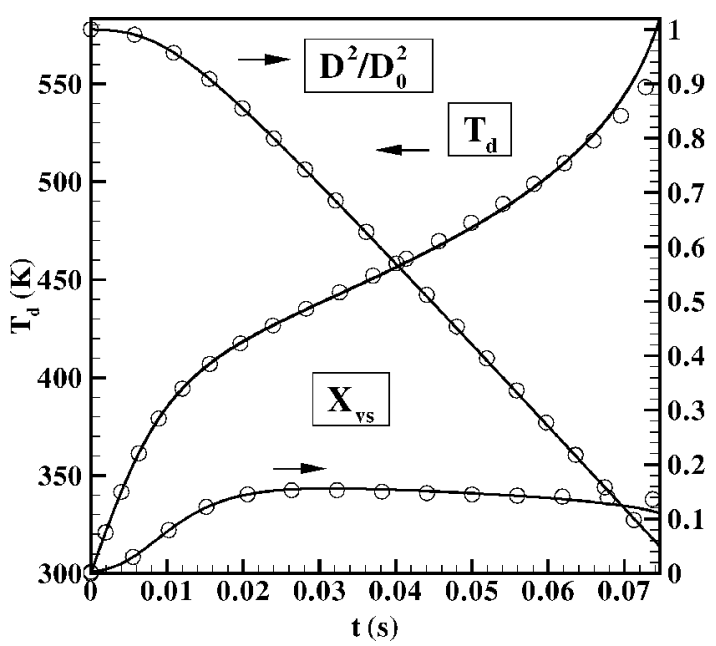

a)

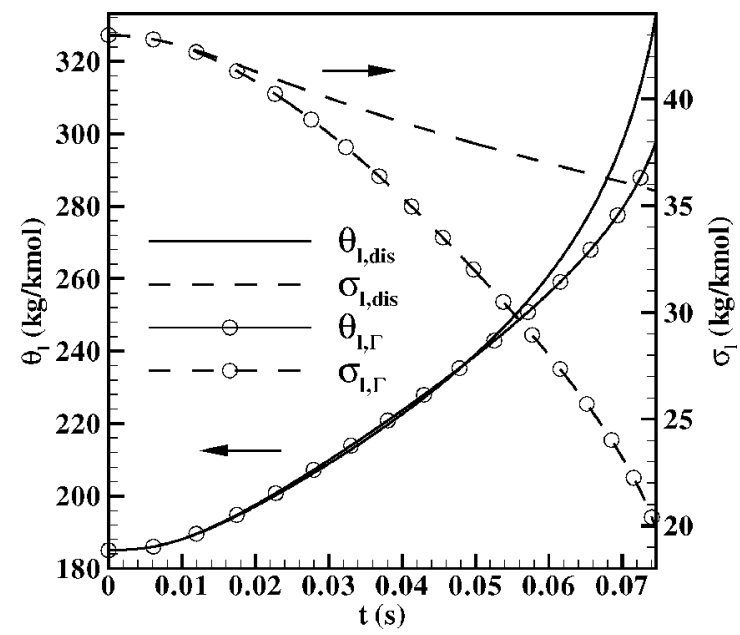

b)

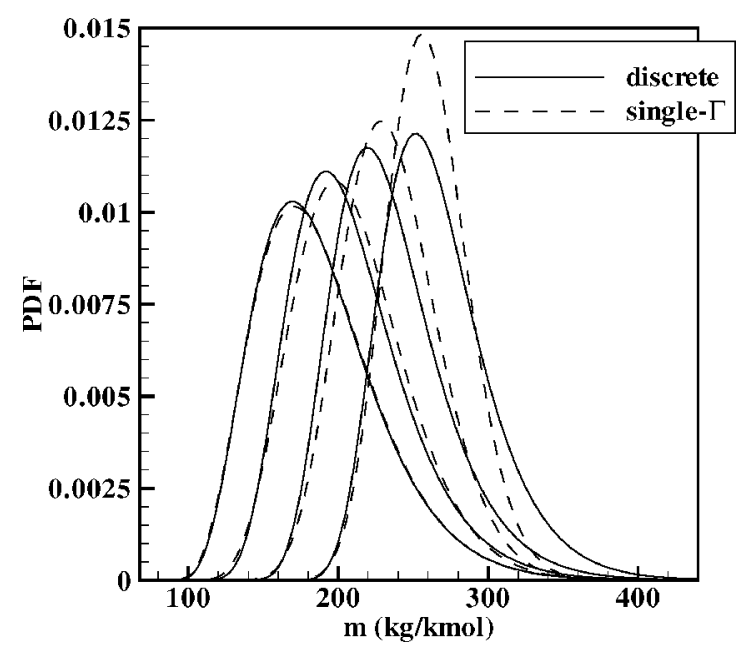

c)

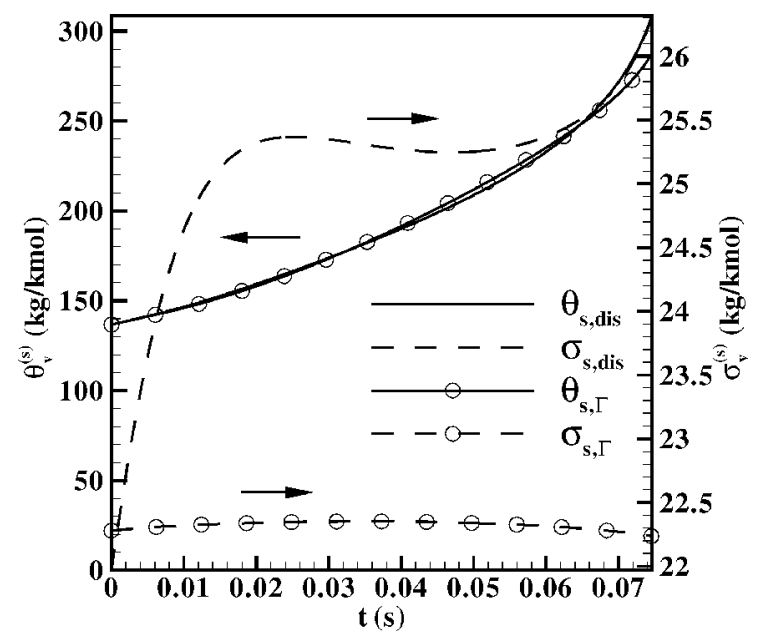

d)

Fig. 2 Evaporation of a diesel fuel drop in a vapor-free environment (run 1, Table 1), discrete model vs the single- $\Gamma$-PDF model: a) drop temperature, relative surface area, and surface vapor mole fraction evolution in time; b) liquid mean molar weight and standard deviation evolution in time; c) PDF at different stages of evaporation, from left to right $90,60,30$, and 10, remaining mass; and d) surface mean molar weight and standard deviation evolution in time. 


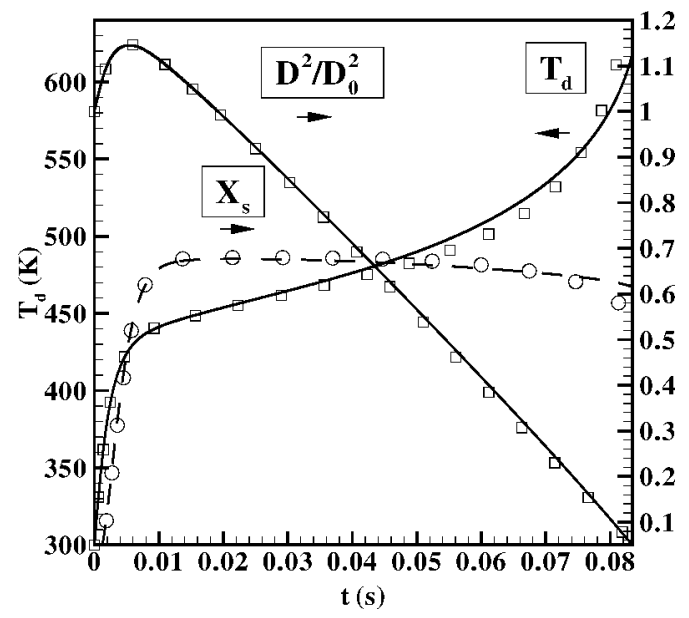

a)

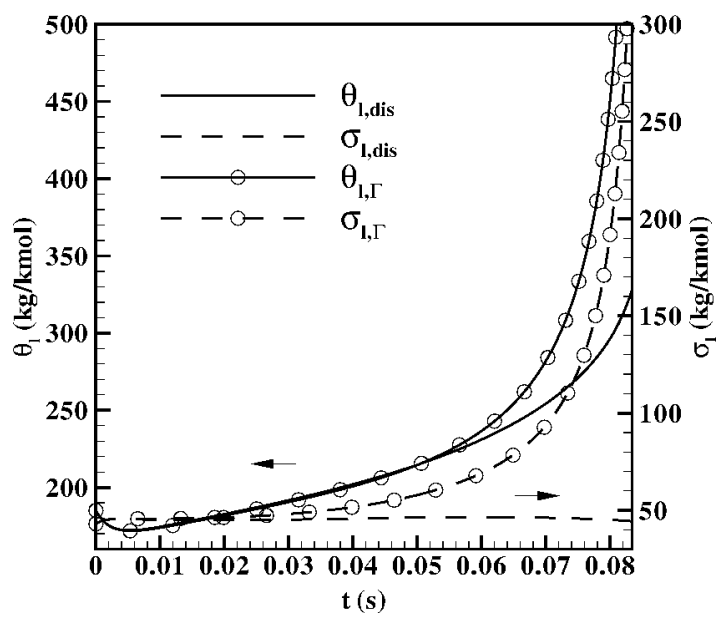

b)

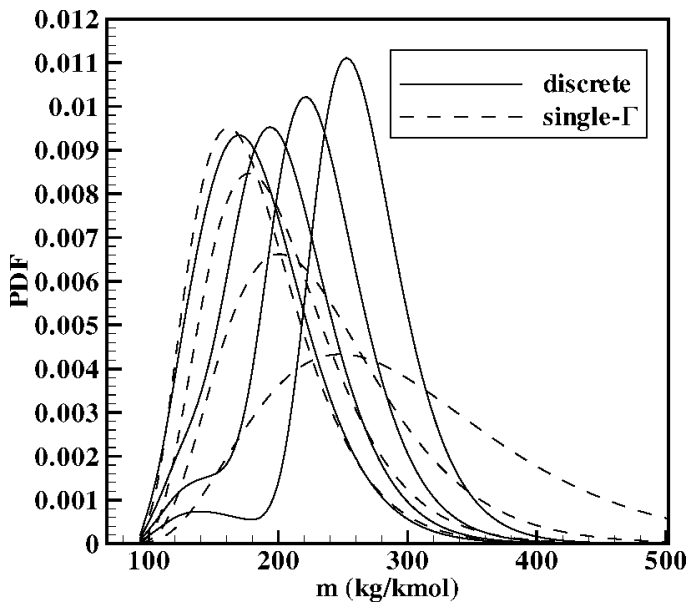

c)

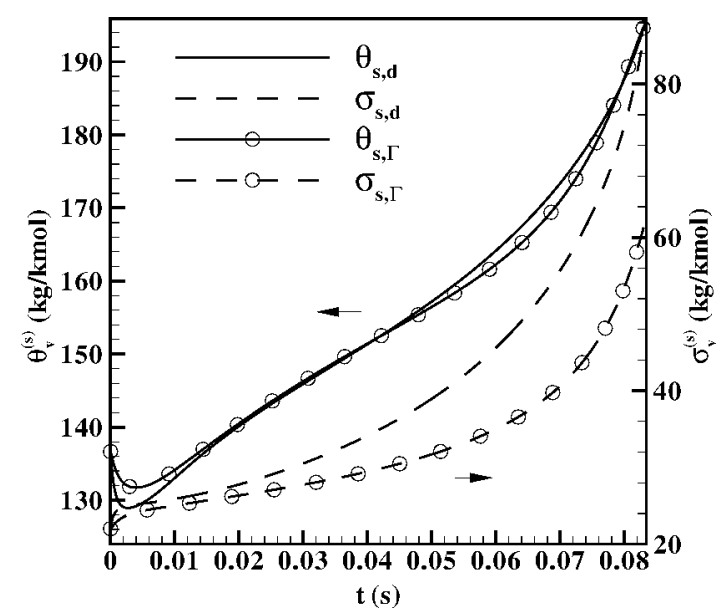

d)

Fig. 3 Evaporation of a diesel fuel drop, discrete model vs the single- - -PDF model (run 2, Table 1): a) drop temperature, relative surface area, and drop surface vapor mole fraction; b) liquid mean molar weight and standard deviation evolution in time; c) PDF at different stages of evaporation, from left to right $90,60,30$, and $10 \%$ remaining mass; and d) surface mean molar weight and standard deviation evolution in time.

visible on this scale) was used to create the database of discretemodel results that served as basis for comparison with the other models.

\section{B. Limitations of the Single- $\Gamma$-PDF Model}

Results from two calculationsillustrate the problems of the single$\Gamma$-PDF model. As a preliminary, consider a drop having an initial diameter $D_{0}=0.1 \mathrm{~mm}$ at an initial temperature $T_{d, 0}=300 \mathrm{~K}$ immersed in surroundings characterized by $T_{g}^{(\infty)}=1000 \mathrm{~K}$ and $Y_{v}^{(\infty)}=0$, as in run 1 listed in Table 1. Results illustrated in Fig. 2 show comparisons between the discrete and the single- $\Gamma$-PDF models. Both models predict the same variation of $D^{2} / D_{0}^{2}$ and $T_{d}$, and $X_{v}^{(s)}$ differs only in the last stages of evaporation (Fig. 2a). In contrast to the traditional single-componentdrop evaporation where $T_{d}$ reaches a steady state, here $T_{d}$ continues to increase because of the linear dependency of the boiling temperature on the mean molar weight. One major difference between the discrete and single- $\Gamma$ PDF models is in the evolution of the liquid composition, shown in Fig. 2b. Whereas there is reasonable agreement between the $\theta_{l}$ values, there are large quantitative discrepancies between the predicted $\sigma_{l}$ values, although both models predict a reduction in $\sigma_{l}$. These discrepancies start early in the drop lifetime and cannot be ignored. The evolutions of the liquid discrete model PDF and the single- $\Gamma$-PDF are shown in Fig. $2 c$ at several stages of the drop lifetime. The PDF from the discrete model is plotted as the envelope of the bar chart that represents the discrete model results. The grad- ual departure of the single- $\Gamma$-PDF from the discrete model PDF is easily observable. Finally, the impact of these discrepancies on the surface vapor composition is shown in Fig. 2d, where it is evident that the single- $\Gamma$-PDF surface vapor standard deviation is smaller by $10 \%$ than that predicted by the discrete model.

In sprays, drops evaporate in an environment that already contains vapor. During MC-fuel drop evaporation, therefore, there is a complete coupling between the evolution of the species from the drop and both the far-field vapor mass fraction and the far-field vapor composition. To understand the behavior of the single- $\Gamma$-PDF under these circumstances, consider the conditions of run 2 listed in Table 1. For run 2, all initial conditions are the same as in run 1, except that $Y_{v}^{(\infty)}=0.82$ with the far-field vapor composition specified as shown in Table 1. Plots equivalent to those of Fig. 2 are presented in Fig. 3. A relatively large amount of vapor in the gas phase induces initial drop net condensation and the drop grows in size, as shown in Fig. 3a. Thereafter, net evaporation begins, during which, following a short transient, the linear $D^{2}$ law is recovered. Initially, $T_{d}$ increases more sharply than in Fig. 2a because the condensed mass adds heat to the drop in the form of latent heat. Similar to the comparison in Fig. 2, there is excellent agreement between the discrete and the single- $\Gamma$-PDF model predictions for $D^{2} / D_{0}^{2}$ and $T_{d}$, and $X_{v}^{(s)}$ differs only after the drop residual mass is less than $15 \%$. Examination of Fig. $3 b$ reveals that the initial condensation results in a decrease in $\theta_{l}$ and a concomitant increase in $\sigma_{l}$, which accounts for the species added to the liquid mixture. Further evaporation results in an increase in $\theta_{l}$ and a decrease in $\sigma_{l}$, as shown by the results 

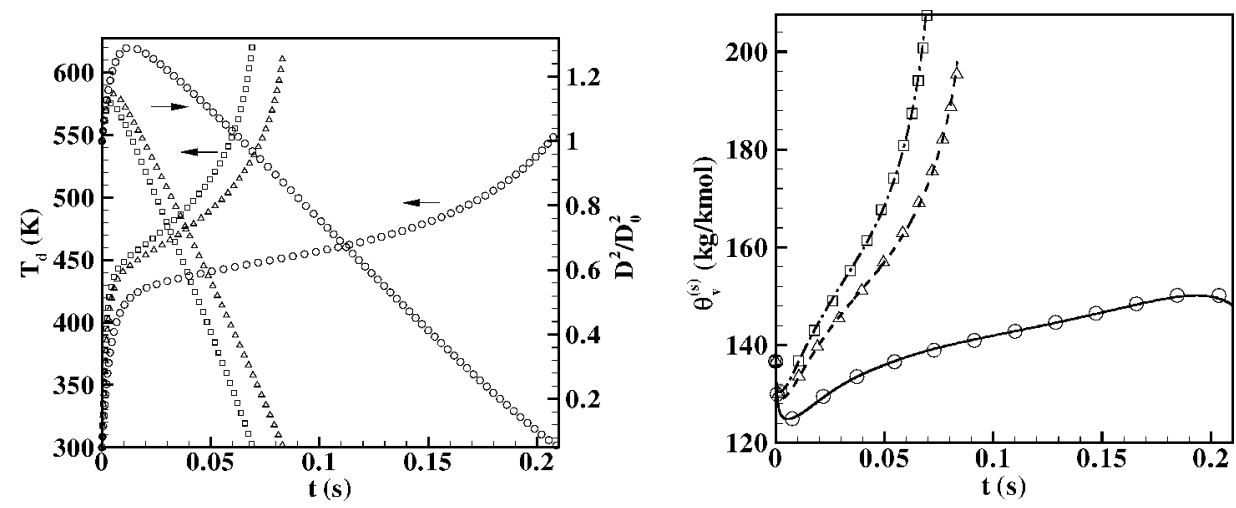

a)

e)
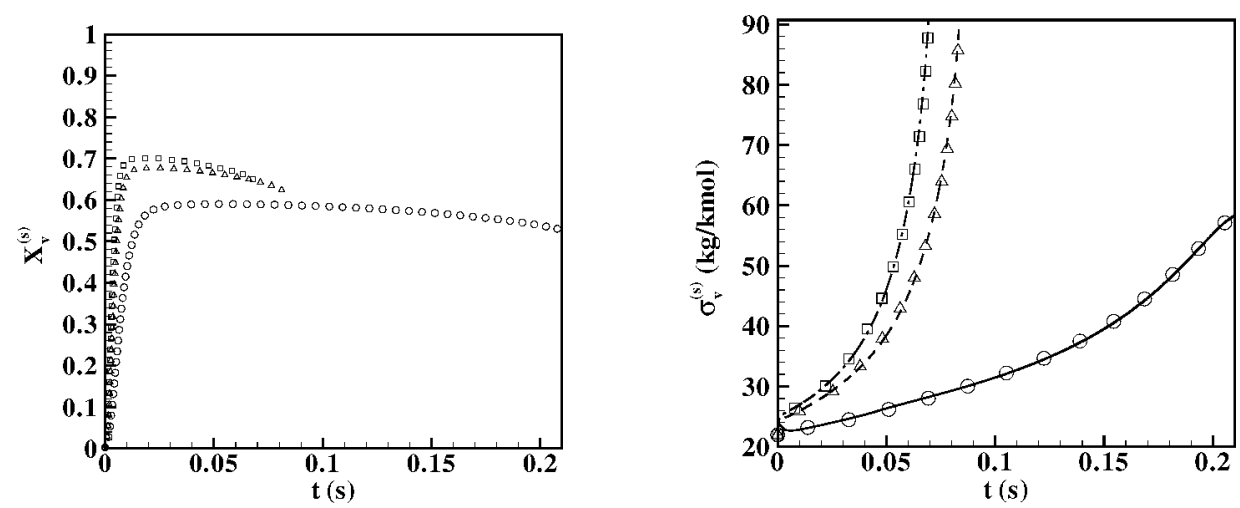

b)

f)
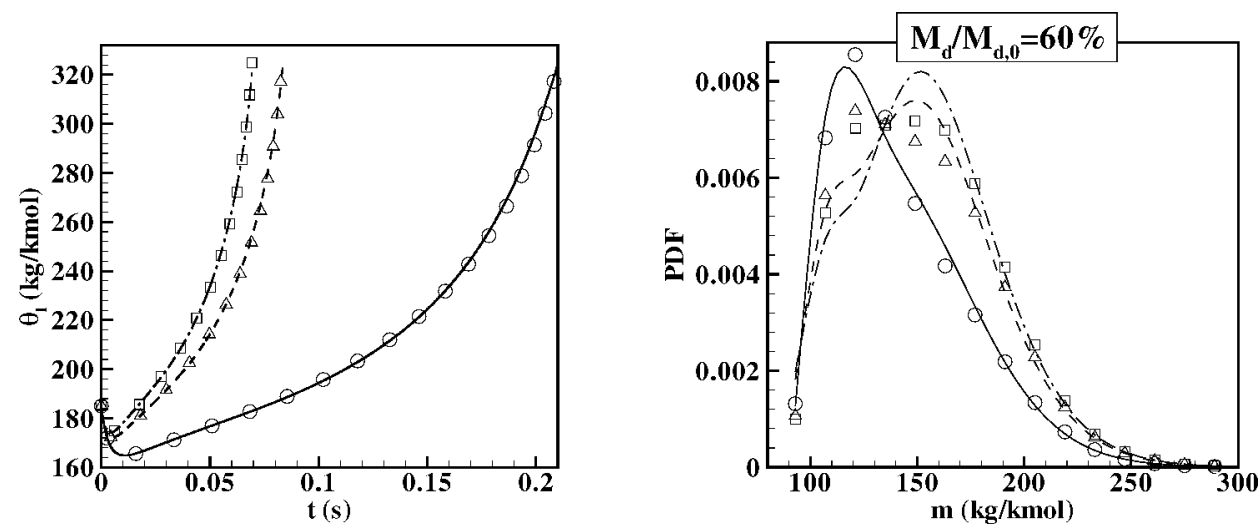

c)

g)
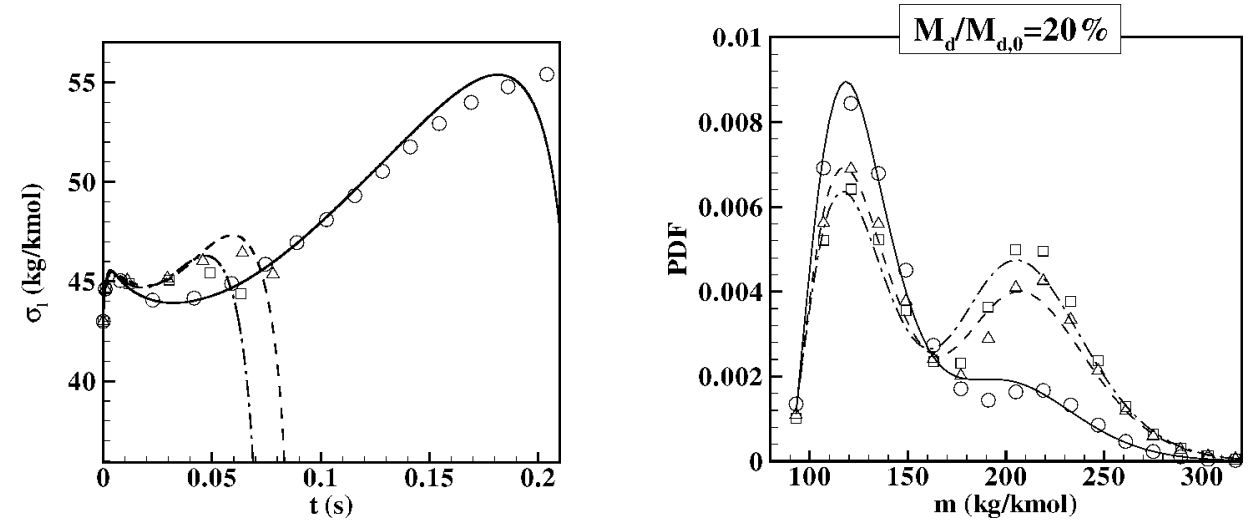

h)

Fig. 4 Diesel drop history for runs 2-4, Table 1: a) drop temperature and relative surface area, b) drop surface vapor mole fraction, c) liquid mean molar weight, d) liquid PDF standard deviation, e) surface composition mean molar weight, f) surface composition PDF standard deviation, g) surface vapor PDFs at $60 \%$ residual drop mass, and $\mathrm{h}$ ) surface vapor PDFs at $20 \%$ residual drop mass: symbols, discrete model; lines, double- $\Gamma$-PDF model; $\longrightarrow$ and $^{\bigcirc}, T_{g}^{(\infty)}=600 \mathrm{~K} ;---$ and $\triangle, T_{g}^{(\infty)}=1000 \mathrm{~K}$; and $-\cdot-$ and $\square, T_{g}^{(\infty)}=1200 \mathrm{~K}$. 
from the discrete model. However, the single- $\Gamma$-PDF results display the opposite behavior in $\sigma_{l}$ in that it increases. Thus, unlike when $Y_{v}^{(\infty)}=0$, now it is no longer only the quantitative values but the qualitative trends that are not captured by the single- $\Gamma$-PDF model. This is easily observable in Fig. 3c, where the discrete model distribution displays another, smaller peak as evaporation proceeds, which the single- $\Gamma$-PDF model inherently cannot replicate. It is the emergence of this second peak that prompted the development of a new model based on the combination of two $\Gamma$-PDFs. Figure $3 d$ shows the discrepancies between the discrete and single- $\Gamma-P D F$ model predictions for the vapor composition.

One noteworthy result of these comparisons is that neither one of $D^{2} / D_{0}^{2}, T_{d}$, or $X_{v}^{(s)}$ evolutions is a good indicator that a model captures the composition aspects of the liquid or the gas. This observation has important implications in that experimental results focusing on these three variables are not adequate to validate models. It is obvious that composition measurements are necessary to determine whether a model is appropriate.

\section{Results from Double- $\Gamma$-PDF Model}

All initial and boundary conditions of the computations are listed in Table 1. Emulating Lippert, ${ }^{20}$ in most test cases the far-field vapor composition is chosen to be the initial equilibrium vapor composition at the drop surface; this is indeed the most likely environment encountered by a newly injected drop because it represents the most volatile components that would have evaporated from already injected drops. The double- $\Gamma$-PDF model evaluation is performed for diesel and gasoline fuels because they represent the two ends of the spectrum in terms of volatility. As stated earlier, $T_{d}, D^{2} / D_{0}^{2}$, and $X_{v}^{(s)}$ predictions are insensitive to the fuel composition, and, thus, hereafter only the discrete model will be shown.

\section{Diesel Fuel}

Temperature variation. Figure $4 \mathrm{a}$ shows $T_{d}$ and $D^{2} / D_{0}^{2}$ for $T_{g}^{(\infty)}=600,1000$, and $1200 \mathrm{~K}$ (runs 4, 2, and 3). In all cases, the drop initially experiences net condensation before net evaporation begins. Although the extent of net condensation evident from the drop growth decreases with increasing $T_{g}^{(\infty)}$, the initial drop growth rate seems independent of $T_{g}^{(\infty)}$. The rate of heat transfer to the drop increases with $T_{g}^{(\infty)}$, and $T_{d}^{g}$ and $X_{v}^{(s)}$ (Fig. 4b) become larger earlier. During the initial net condensation, $X_{v}^{(s)}$ becomes eventually larger than $X_{v}^{(\infty)}$ but a slight decline occurs during net evaporation. Comparisons between the discrete model and double- $\Gamma$-PDF predictions are presented in Figs. $4 \mathrm{c}-4 \mathrm{f}$ for $\theta_{l}, \sigma_{l}, \theta_{v}^{(s)}$, and $\sigma_{v}^{(s)}$. The agreement between the two models is very good to excellent. Particularly, $\theta_{v}^{(s)}$ and $\sigma_{v}^{(s)}$, which are the quantities of interest in predicting the composition of the gas phase, are very accurately predicted. During net condensation, $\sigma_{l}$ increases and $\theta_{l}$ decreases due to the addition of the lighter, far-field species; as net evaporation initiates, the lighter species leave the drop, resulting in the reverse trend.

Finally, Figs. $4 \mathrm{~g}$ and $4 \mathrm{~h}$ show a comparison between the discrete model and the double- $\Gamma$-PDF of the surface vapor at two times corresponding to a residual liquid mass of 60 and 20\%, respectively. At the smallest $T_{g}^{(\infty)}$ and early in the drop lifetime, the discrete model PDF visibly has a single peak, which is located in the lower- $m$ regime of the double- $\Gamma-P D F$, consistent with the fact that during slow drop heating the more volatile components are first released from the drop. Another, minor peak, which was barely evident during the early drop lifetime, develops in the larger$m$ regime during the later stages of the drop life. For the larger values of $T^{(\infty)}$ and at $60 \%$ of residual liquid mass, a broad discretemodel PDF-peak develops at intermediate $m$, which transforms into a dominant lower- $m$ and a minor higher- $m$ peak later during the drop lifetime. The physical explanation for this behavior is that at higher ambient temperature, the less volatile components may also evaporate because there is a larger heat flux into the drop leading to a higher drop temperature. When the size of the two peaks is compared, the longest net condensation period, which occurs at the smallest $T_{g}^{(\infty)}$ naturally leads to the largest peak at the lower- $m$ regime of the double- $\Gamma$-PDF. The double- $\Gamma$-PDF captures the discrete model both in the early and later stage of the drop lifetime and, thus, reproduces the differential species evaporation as a function of $T_{g}^{(\infty)}$. The importance of the dominant lighter components in the gas composition highlights the necessity of the double- $\Gamma-P D F$ representation.

To understand the relationship between the lower- $m$ peak in the surface vapor and liquid PDFs, consider the PDF of the liquid composition shown in Fig. 5 at the two times corresponding to a residual

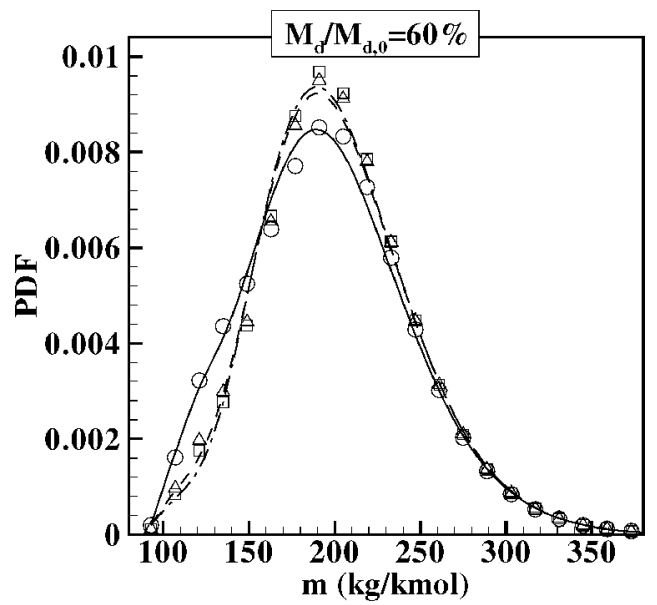

a)

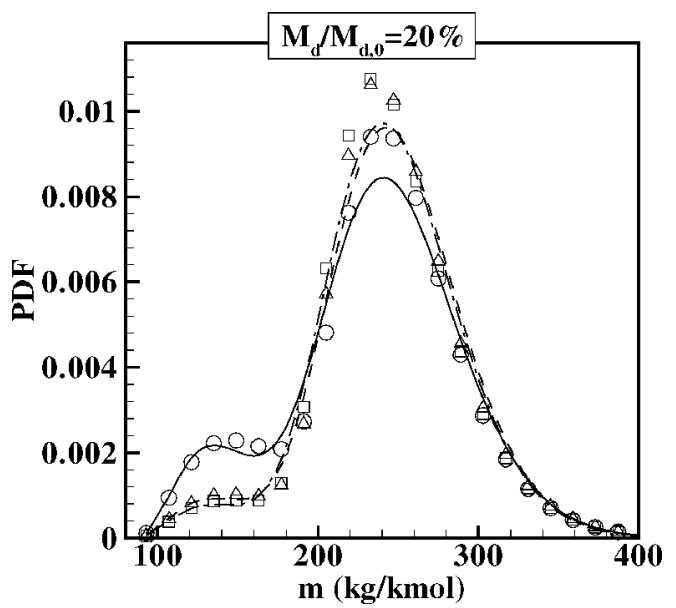

b)

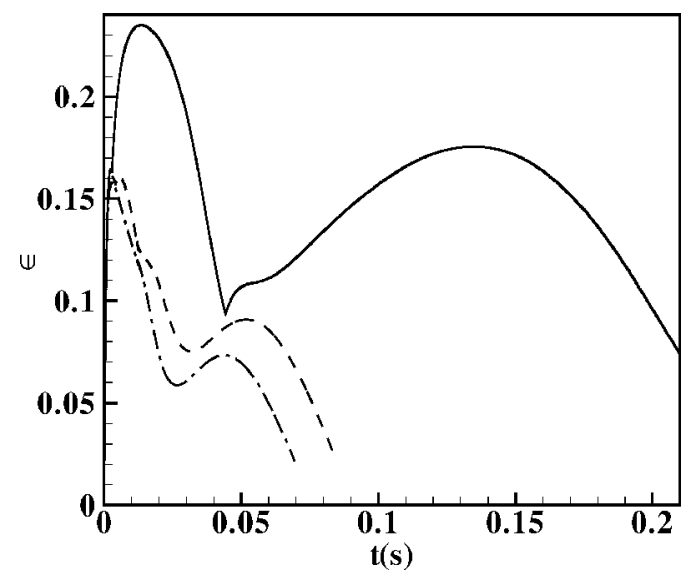

c)

Fig. 5 Characteristics of the liquid double- $\Gamma-P D F$ for runs 2-4: a) PDF at $60 \%$ of the drop initial mass, b) PDF at $20 \%$ of the drop initial mass, and c) $\epsilon$ timewise evolution. 


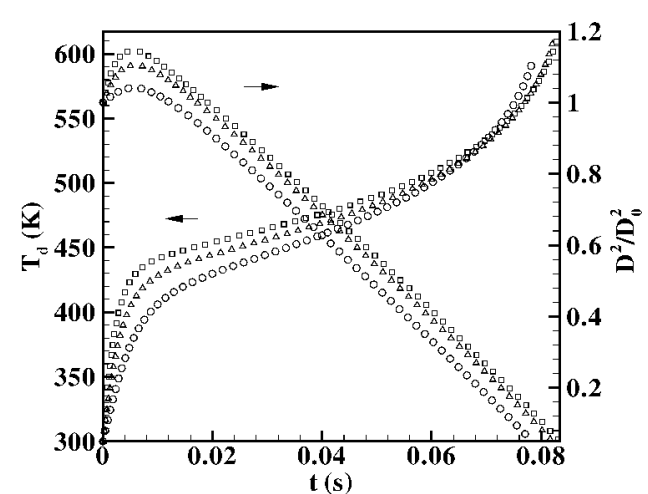

a)

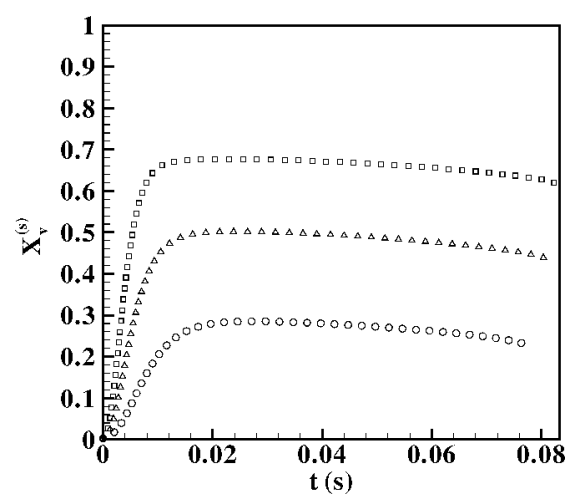

b)

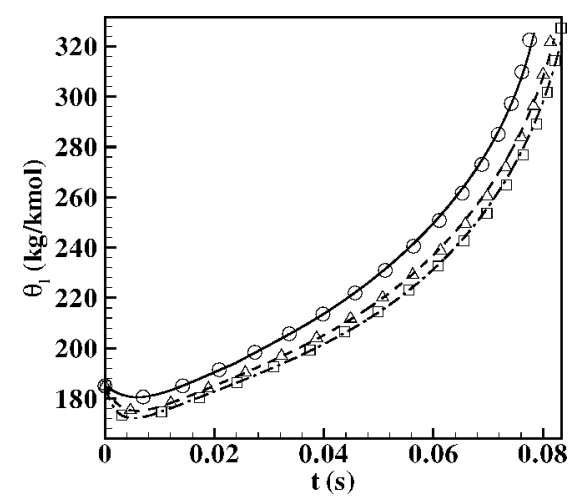

c)

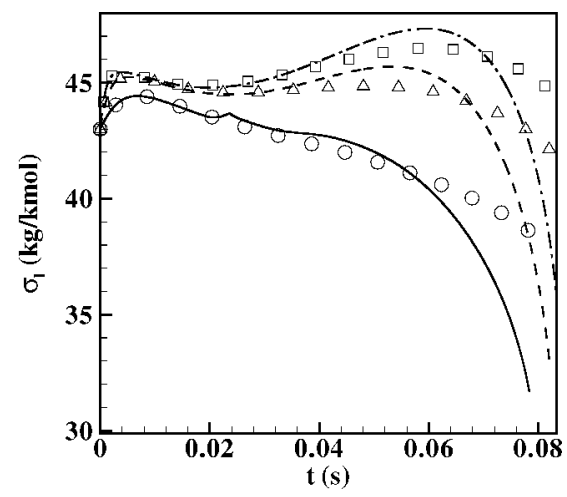

d)

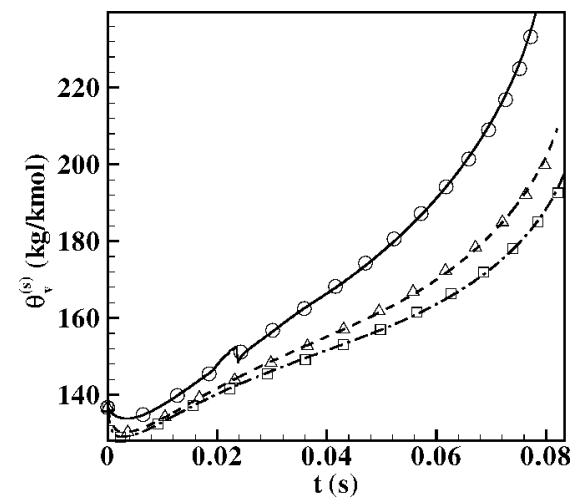

e)

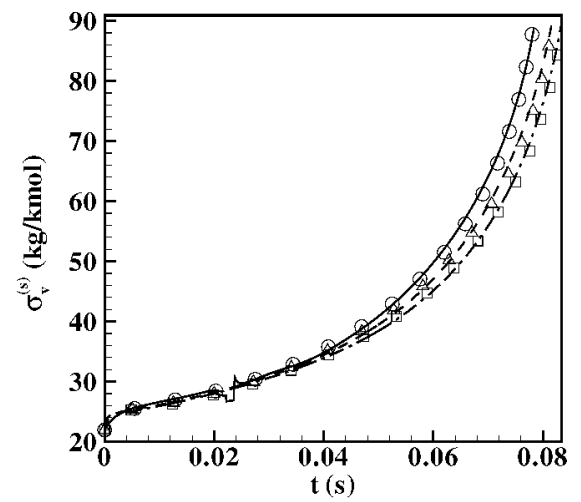

f)

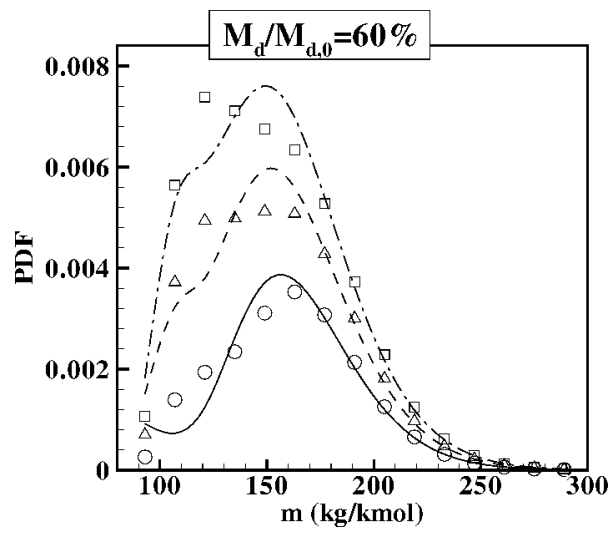

g)

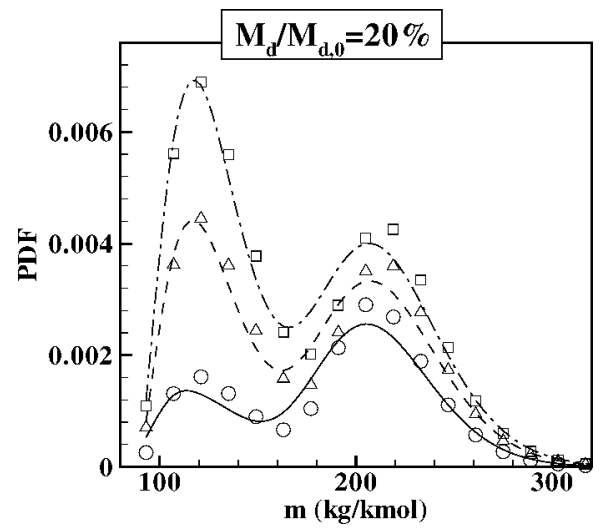

h)

Fig. 6 Diesel drop history for runs 2, 5, and 6: a) drop temperature and relative surface area, b) drop surface vapor mole fraction, c) liquid mean molar weight, d) liquid PDF standard deviation, e) surface composition mean molar weight, $f$ ) surface composition PDF standard deviation, $g$ ) surface vapor PDFs at $60 \%$ residual drop mass, and $h$ ) surface vapor PDFs at $20 \%$ residual drop mass: symbols, discrete model; lines, double- $\Gamma$-PDF model; and $^{\circ}, X_{v}^{(\infty)}=0.1,---$ and $\triangle, X_{v}^{(\infty)}=0.3$; and - - - and $\square, X_{v}^{(\infty)}=0.5$. 


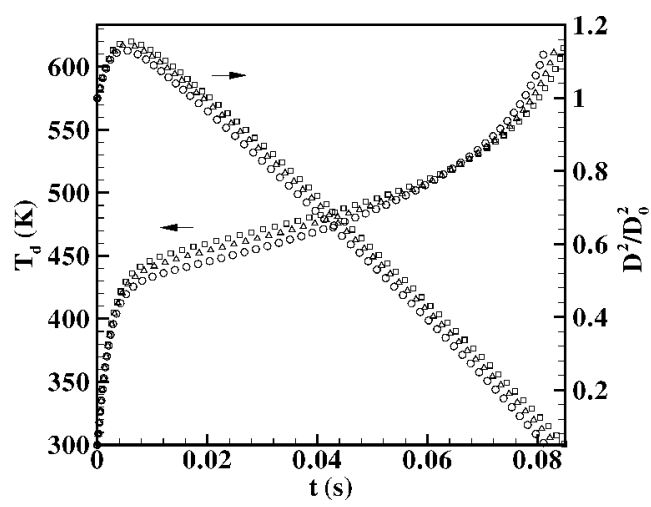

a)

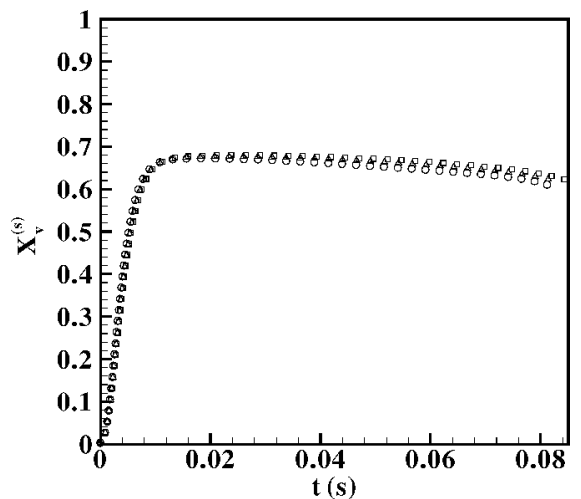

b)

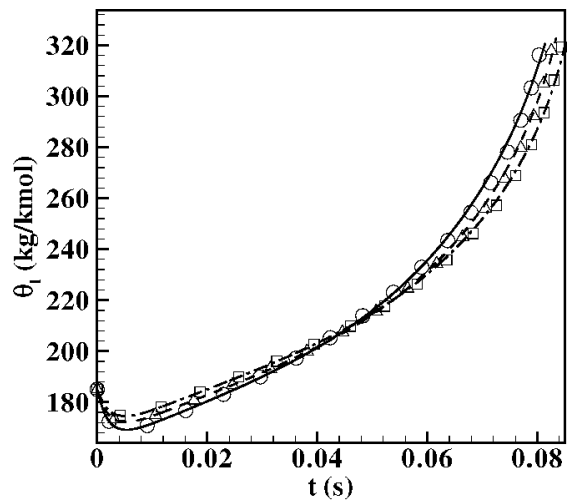

c)

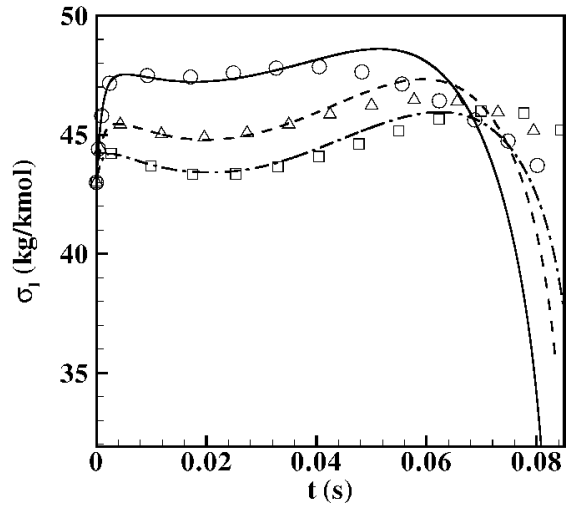

d)

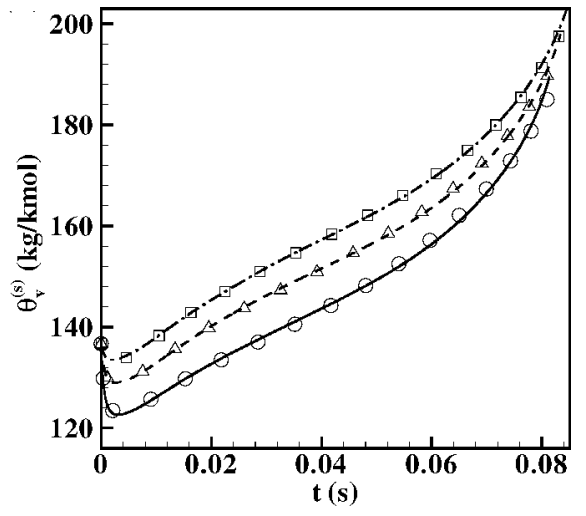

e)
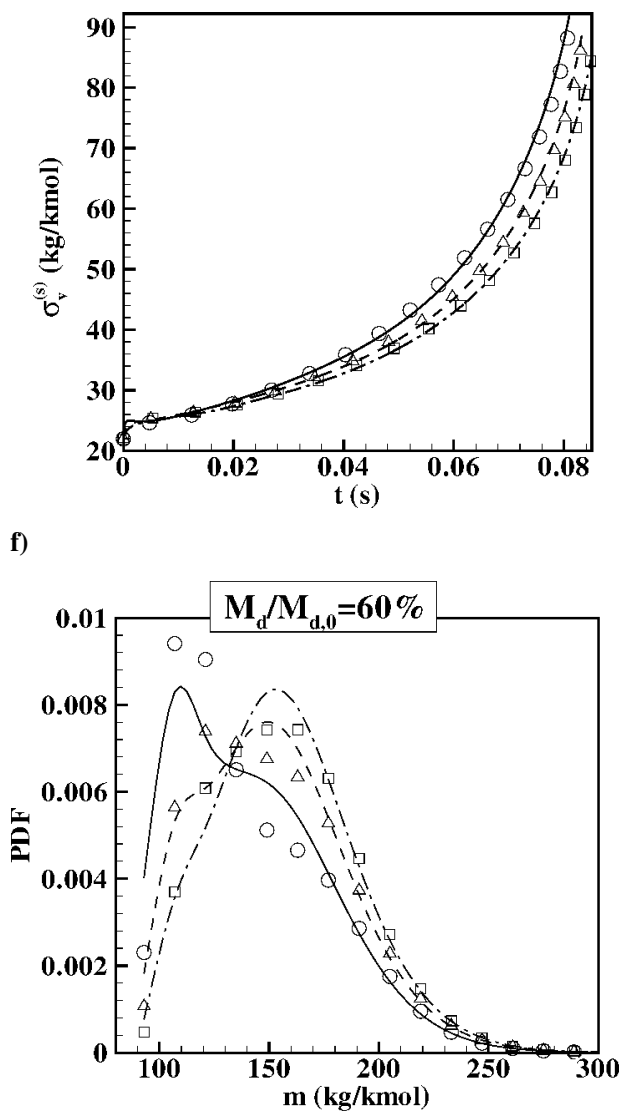

g)

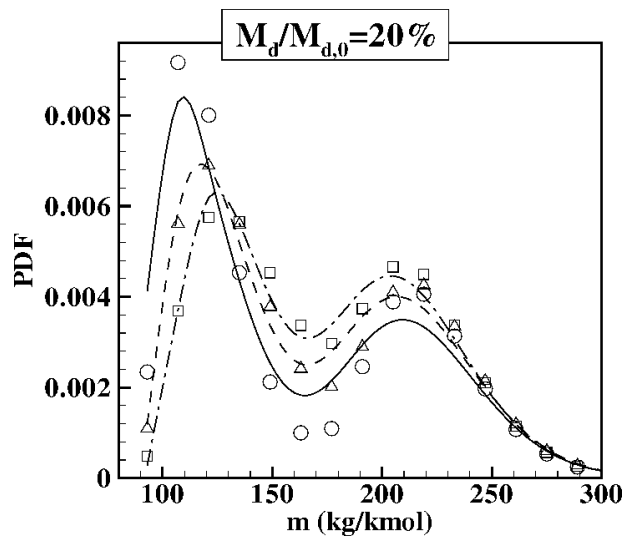

h)

Fig. 7 Diesel drop history for runs 2, 7, and 8: a) drop temperature and relative surface area, b) drop surface vapor mole fraction, c) liquid mean molar weight, d) liquid PDF standard deviation, e) surface composition mean molar weight, f) surface composition PDF standard deviation, g) surface vapor PDFs at $60 \%$ residual drop mass, and $\mathrm{h}$ ) surface vapor PDFs at $20 \%$ residual drop mass: symbols, discrete model; lines, double- $\Gamma$-PDF model; $\longrightarrow$ and $^{\bigcirc}, \theta_{v}^{(\infty)}=120 \mathrm{~kg} / \mathrm{kmol}$ and $\sigma_{v}^{(\infty)}=18 ;---$ and $\triangle, \theta_{v}^{(\infty)}=131.3 \mathrm{~kg} / \mathrm{kmol}$ and $\sigma_{v}^{(\infty)}=24.4 ;$ and $-\cdot-$ and $\square, \theta_{v}^{(\infty)}=140 \mathrm{~kg} / \mathrm{kmol}$ and $\sigma_{v}^{(\infty)}=28$. 
liquid mass of $60 \%$ (Fig. 5a) and $20 \%$ (Fig. 5b). At $60 \%$ of the residual mass, the PDFs display generally a single peak, although a nascent lower- $m$ protuberance is emerging. At $20 \%$ of the residual mass, the lower- $m$ regime peak is evident in all PDFs, and its magnitude increases with decreasing $T_{g}^{(\infty)}$, that is, with increasing initial net condensation period, which lead to the largest peak in the lower- $m$ regime for the surface vapor composition PDF. This difference between the lowest $T_{g}^{(\infty)}$ PDF and those at larger $T_{g}^{(\infty)}$ is consistent with the $\sigma_{l}$ behavior and excellently captured by the double- $\Gamma$-PDF at all stages of the drop lifetime. Thus, although the surface vapor composition is eventually dominated by the low $m$ species, the liquid composition still peaks at a relatively large $m$ during the earlier part of the drop lifetime and is dominated by the higher components during the later part of the drop lifetime. The fact that relatively light components remain inside the drop close to the end of the drop life is noticeable and is the result of eventual saturation. The double- $\Gamma$-PDF representation faithfully replicates the discrete model.

Because the evolution from the initial condition, single- $\Gamma-P D F$ to the double- $\Gamma$-PDF, is represented in Eq. (12) by $\epsilon$, in Fig. 5c we show its time variation. At the lowest $T_{g}^{(\infty)}, \epsilon$ displays a sharp increase from its initial null value then decreases, and after a kink, it evolves with a continuingnonmonotonicbehavior. The same general behavior, but with less dramatic variations is exhibited at the two other $T_{g}^{(\infty)}$ values, and $\epsilon$ decreases with increasing $T_{g}^{(\infty)}$. Although $\epsilon$ is small with respect to unity (here, $\epsilon \in[0.02,0.27]$ ), it allows the development of the minor, lower- $m$ peak in the liquid PDF, which translates into the dominant, lower- $m$ peak in the surface vapor PDF.

Effect of the far-field mole fraction. To explore the effect of $X_{v}^{(\infty)}$, we compared results from runs 2,5 , and 6 . The net condensation rate in the early stages of the drop lifetime increases with $X_{v}^{(\infty)}$, as shown in Fig. 6a, leading to larger drops before net evaporation begins. Consistent with the higher net condensation rate, the initial $\mathrm{d} T_{d} / \mathrm{d} t$ is larger as well. Initially, $X_{v}^{(s)}$ increases during the net condensation period, but an eventual asymptotic behavior develops (Fig. 6b). The agreement of the double- $\Gamma$-PDF with the discrete model is excellent for $\theta_{l}, \theta_{v}^{(s)}$, and $\sigma_{v}^{(s)}$ and very good to fair for $\sigma_{l}$, as shown in Figs. 6c-6f. The small glitch in the $\theta_{v}^{(s)}$ and $\sigma_{v}^{(s)}$ curves for $X_{v}^{(\infty)}=0.1$ corresponds to the time when $\epsilon$ experiences a sharp change in curvature (for example, Fig. 5c), but the model is robust enough to overcome this small, local timewise discontinuity and the computation quickly recovers and continues to lead to excellent agreement with the discrete model. We note that the excellent agreement in $\theta_{v}^{(s)}$ and $\sigma_{v}^{(s)}$ is a key element for robust two-phase flow computations.

Comparison of the surface vapor double- $\Gamma$-PDF with the discrete model PDF at 60 and $20 \%$ of the initial mass is presented in Figs. $6 \mathrm{~g}$ and $6 \mathrm{~h}$. At $60 \%$ residual mass, a minor peak in the discrete distribution is already visible at the lower $X_{v}^{(\infty)}$, which is fairly well replicated by the double- $\Gamma$-PDF. As $X_{v}^{(\infty)}{ }^{v}$ increases and at $60 \%$ residual mass, the two peaks first merge into a broader intermediate- $m$ peak, which becomes a single lower- $m$ peak at the largest $X_{v}^{(\infty)}$. By $20 \%$ residual mass, the lower- $m$ peak becomes dominant except at the lowest $X_{v}^{(\infty)}$, and all double- $\Gamma$-PDFs reproduce the result of the discrete model with remarkable accuracy. The lower- $m$ peak increases with $X_{v}^{(\infty)}$, which together with the fact that it also increased with decreasing $T_{g}^{(\infty)}$ suggests that it is due to the condensation process. Such condensation is inevitable in sprays where drops are transported in regions of different temperature and composition, and the capturing of this physics is considered essential to the model utilization under spray conditions.

Comparison of the respectiveeffects of $T_{g}^{(\infty)}$ and $X_{v}^{(\infty)}$ shows that the former has a much larger impact on the vapor composition. In fact, $\sigma_{v}^{(s)}$ is almost independent of $X_{v}^{(\infty)}$.

Effect of the ambient composition. In Fig. 7, we compare results from runs 2, 7 and 8 to assess the influence of $\theta_{v}^{(\infty)}$ and $\sigma_{v}^{(\infty)}$ on the predictions. Apparently, neither $T_{d}$ or $D^{2} / D_{0}^{2}$ (Fig. 7a) nor $X_{v}^{(s)}$ (Fig. 7b) are sensitive to the far-field composition. What matters for

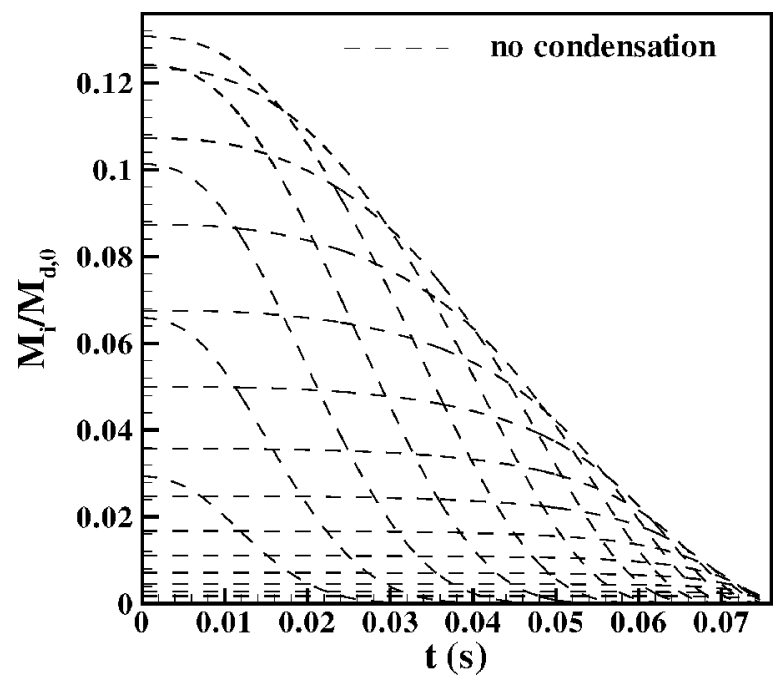

a)

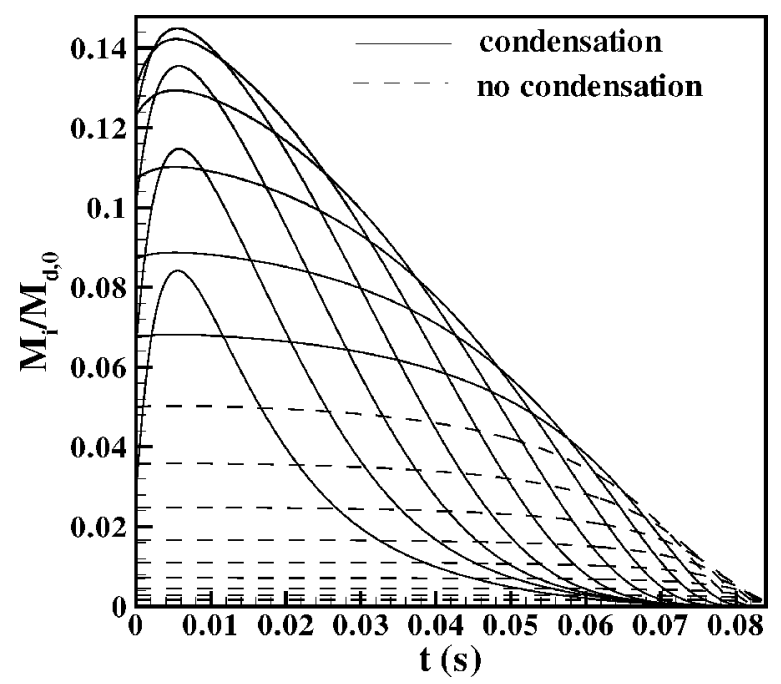

b)

Fig. 8 Timewise evolution of nondimensionalized species partialmass for a) vapor-free environment (run 1) and b) $X_{v}^{(\infty)}=0.5$, $\theta_{v}^{(\infty)}=131.3 \mathrm{~kg} / \mathrm{kmol}$ and $\sigma_{v}^{(\infty)}=24.4($ run 2$)$.

the magnitude of these variables is the far-field vapor molar fraction but not the vapor composition. This explains why these parameters are insensitive to the model used (single- $\Gamma-\mathrm{PDF}$ vs double- $\Gamma-\mathrm{PDF}$ ). The drop net growth rate is the same for all three runs, and only a small increase in $D^{2} / D_{0}^{2}$ and in the duration of condensation is discerniblewith increasing $\theta_{v}^{(\infty)}$ and $\sigma_{v}^{(\infty)}$. As for $\theta_{l}, \theta_{v}^{(s)}$ and $\sigma_{v}^{(s)}$, the agreement of the double- $\Gamma$-PDF with the discrete model is excellent (Figs. 7c-7f), whereas $\sigma_{l}$ (Fig. 7d) is very well predicted during most of the drop lifetime. Although it would be desirable to have a better replication of $\sigma_{l}$ by the double- $\Gamma-\mathrm{PDF}$, we reiterate that it is the prediction of $\theta_{v}^{(s)}$ and $\sigma_{v}^{(s)}$ that is important in spray calculations for which this model is being developed.

The suggestion that the lower- $m$ peak in the double- $\Gamma$-PDF originates from condensation is strengthened by the plots of the surface vapor PDF at 60\% (Fig. 7g) and 20\% (Fig. 7h) residual mass. At 60\% residual mass, all PDF exhibit two peaks, and for the two smaller $\theta_{v}^{(\infty)}$, the magnitude of the lower- $m$ peak is already dominant. During the entire drop evolution, the magnitude of the lower- $m$ peak depends inversely on the initial liquid-PDF mean. Further adding to the argument that the condensation process is responsible for the lower- $m$ peak is that the molar weight composition of that peak is similar to that of the far field. The agreement of the double- $\Gamma$-PDF with the discrete model is very good.

Because all results seem to indicate that the minor peak is a consequence of the condensation process, it is of interest to follow the 


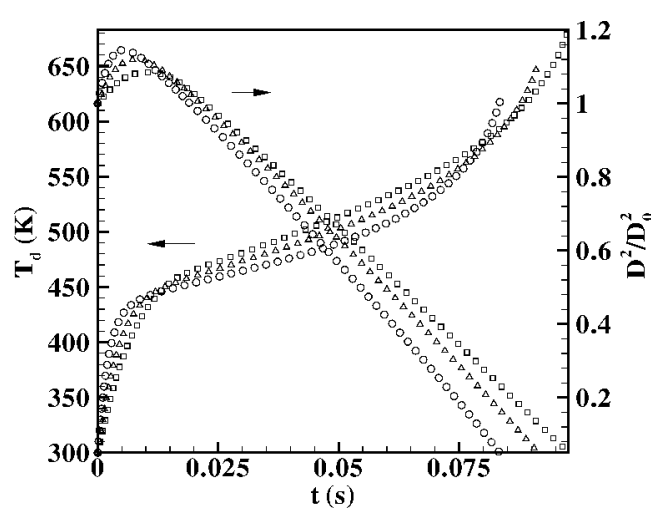

a)

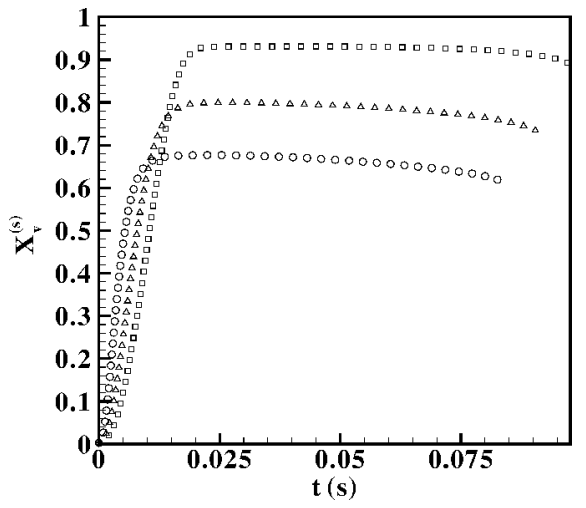

b)

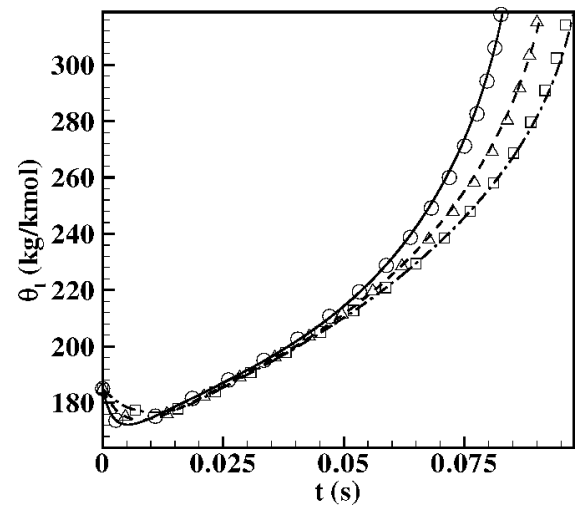

c)

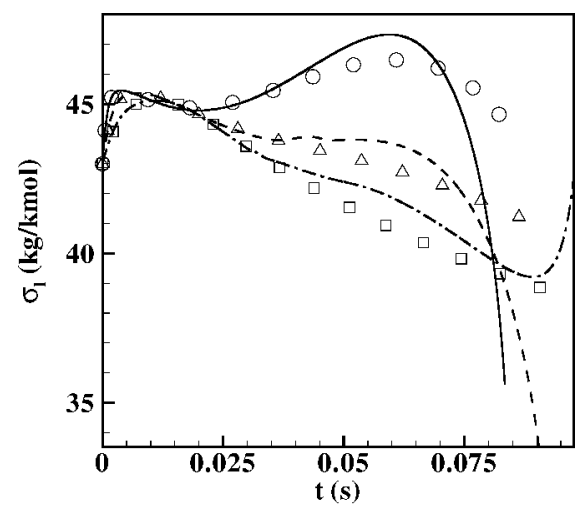

d)

Fig. 9 Diesel drop history for runs 2, 9, and 10: a) drop temperature and relative surface area, b) drop surface vapor mole fraction, c) liquid mean molar weight, d) liquid PDF standard deviation, e) surface composition mean molar weight, f) surface composition PDF standard deviation, g) surface vapor PDFs at $60 \%$ residual drop mass, and h) surface vapor PDFs at $20 \%$ residual drop mass: symbols, discrete model; lines, double- $\Gamma$-PDF model; e)
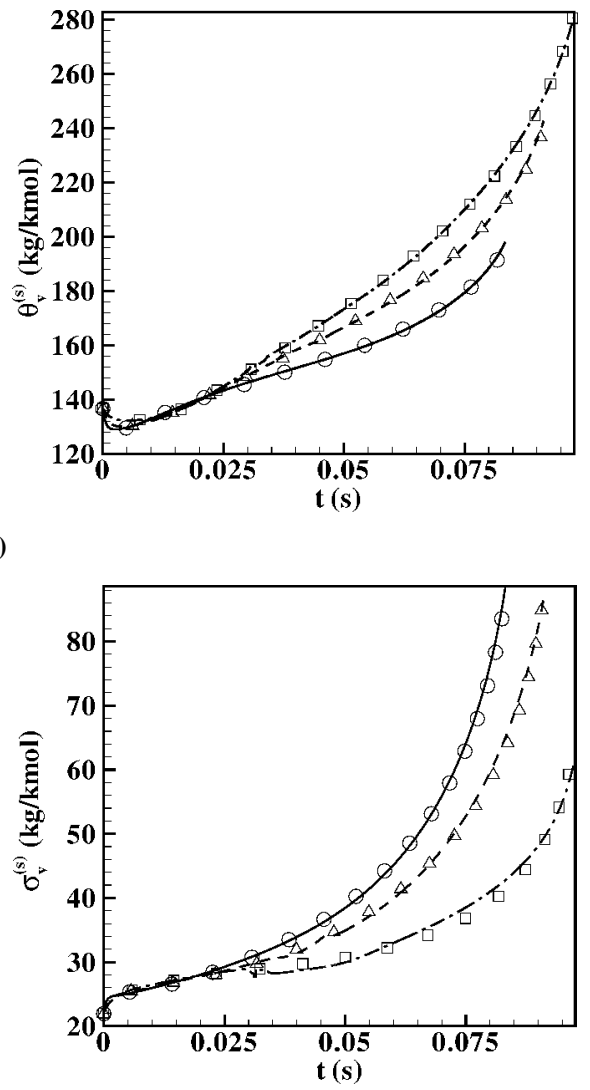

f)

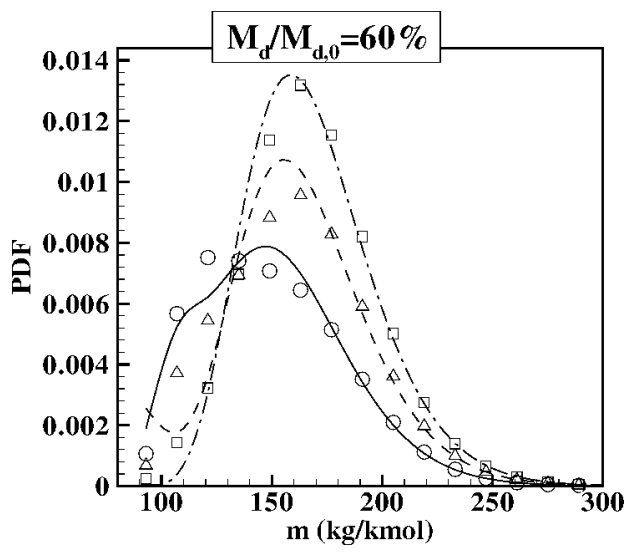

g)

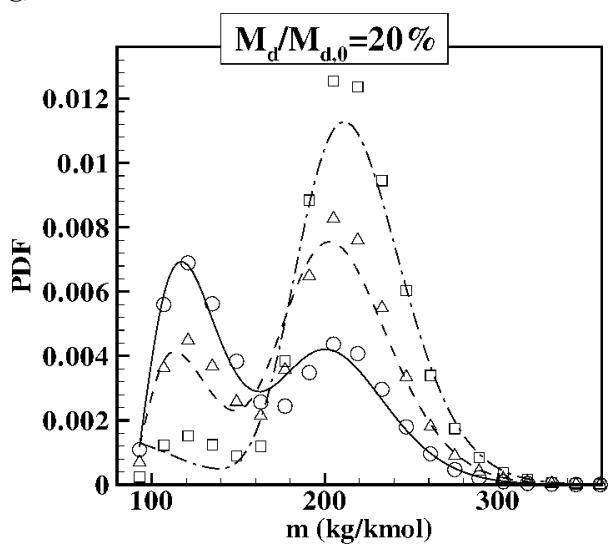

h) 
history of $M_{i} / M_{d, 0}$ associated with a specific $m$. These quantities computed from the discrete model for two different far-field conditions are shown in Fig. 8. In a $X_{v}^{(\infty)}=0$ environment(run 1, Fig. 8a), $M_{i} / M_{d, 0}$ monotonically decreases, and the lighter components disappear earlier from the drop. In an environment with $X_{v}^{(\infty)} \neq 0$ (run 2, Fig. 8b), there are species of molar weight much larger than $\theta_{v}^{(\infty)}$, whose $M_{i} / M_{d, 0}$ decreases monotonically, and species of molar weight comparable to $\theta_{v}^{(\infty)}$, whose $M_{i} / M_{d, 0}$ initially increases, which corresponds to condensation, reach a peak, and then decrease, which corresponds to evaporation. The purpose of this examination is to underline the fate of different species according to how their molar weight compares to the composition of the far field and also to highlight that the drop evolution is the combined manifestation of these different histories. This is precisely where lies the difficulty in developing an appropriate representation of MC-fuel drop evaporation using a small number of species equations.

Gas Lewis number variation. Lewis number effects (runs 2, 9, and 10) are shown in Fig. 9. As the Lewis number increases, the characteristic time for mass diffusion becomes larger with respect to that of heat diffusion in the gas phase. This means that condensation is delayed with increasing Lewis number, which in turn leads to a lower rate of $T_{d}$ increase (Fig. 9a) corresponding to the smaller and delayed drop growth (Fig. 9a) and to a delayed achievement of steady-state evaporation. Thus, drops in a gas having a smaller Lewis number grow faster and to larger size because the initial net condensation is more effective, and they evaporate slightly faster. The much larger rate of increase in $X_{v}^{(s)}$ for the smallest Lewis number (Fig. 9b) portrays the faster condensation, and the earlier reaching of an asymptotic behavior is consistent with an earlier steady state. The steady-state value of $X_{v}^{(s)}$ is larger with increasing Lewis number because the increased mass diffusion time means that the evaporated species tend to stay longer at the surface.

This physical picture is confirmed by Figs. $9 \mathrm{~d}-9 \mathrm{f}$. We note that for all $\theta_{l}, \theta_{v}^{(s)}$, and $\sigma_{v}^{(s)}$ the double- $\Gamma$-PDF results are in excellent agreement with those of the discrete model, and for $\sigma_{l}$ the agreement is very good up to a residual mass of $9 \%$, after which a deterioration is observed at the lower Lewis number. Here $\theta_{l}$ varies inversely with Lewis number, but $\theta_{v}^{(s)}$ decreases with decreasing Lewis number, although the relative variation of $\theta_{l}$ and $\theta_{v}^{(s)}$ is considerably smaller than that in Lewis number, showing that the prediction of this quantity is relatively insensitive to the uncertainties associated with the exact knowledge of $\mathcal{D}_{\text {eff }}$. Both $\sigma_{l}$ and $\sigma_{v}^{(s)}$ are decreasing functions of Lewis number, showing that liquid mixtures have fewer species with increasing Lewis number.

Examination of the surface vapor PDFs at $60 \%$ residual mass (Fig. 9g) shows in all cases the initiation of a minor peak that is located at smaller $m$ with increasing Lewis number. The distribution is wider with decreasing Lewis number. By $20 \%$ residual mass (Fig. 9h), the lower- $m$ peak has become dominant only for the smallest Lewis number value, indicating the retention of the enhanced effect of the lower- $m$ species seen earlier in the lifetime. The agreement of the double- $\Gamma$-PDF with the discrete model is very good for largest Lewis number and excellent for the smallest Lewis number.

\section{Gasoline}

Results similar to those for the diesel study were obtained for gasoline (Table 1); for brevity, these results are not illustrated. Although qualitatively the results of runs 11-13 are similar to those for diesel, here the net condensation time and resulting drop growth play a more reduced role. This explains the opposite initial behavior of $\theta_{l}$ and $\sigma_{l}$ compared to diesel in that for gasoline they both decrease initially, indicating an immediate evaporation of light components. There is again an general excellent agreement between the discrete and double- $\Gamma$-PDF models.

Figure 10 (run 11) shows the gasoline equivalent of Fig. 8b, which was for diesel (run 2), enabling a comparison of the dis-

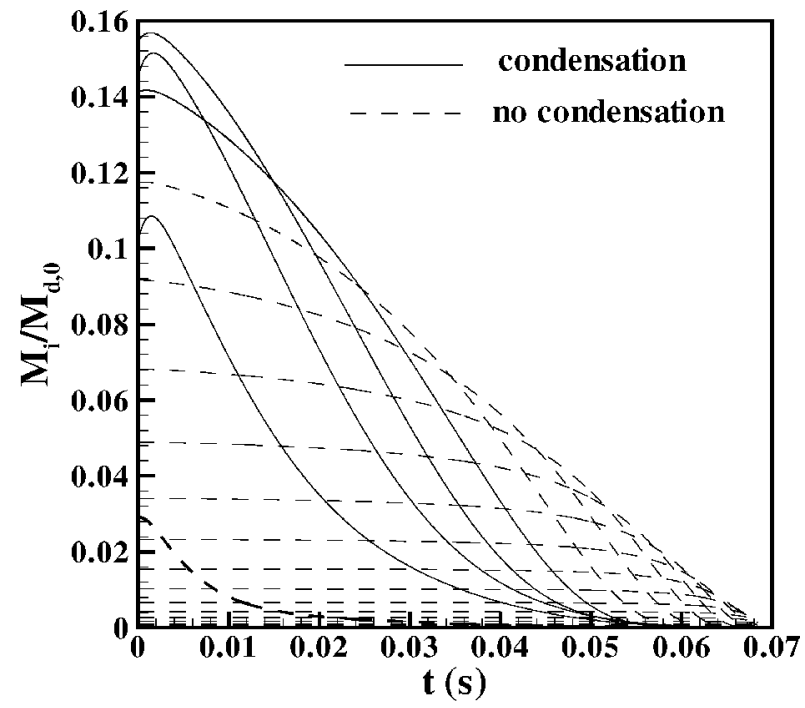

Fig. 10 Timewise evolution of the nondimensional species partialmass calculated according to the discrete model applied to gasoline (run 11).

crete model predictions for the $M_{i} / M_{d, 0}$ time evolution. The calculations are performed for gasoline and diesel at same $T_{g}^{(\infty)}$, $X_{v}^{(\infty)}$, and Lewis number. The different behavior of the two fuels is clearly apparent. For diesel (Fig. 8b) there is a strong initial condensation of many species, whose mass in the drop increases substantially, peaks, and eventually decreases. In contrast, the few species that condense initially in gasoline (Fig. 10) do so only very slightly before evaporating very quickly. The fact that the double- $\Gamma$-PDF can replicate the behavior of the two fuels quite accurately indicates that it has an excellent potential to differentiate between the comportment of real fuels according to their composition.

When varying the far-field mole fraction (runs 11, 14, and 15), the trends are similar to the diesel results, although, comparatively, here $X_{v}^{(\infty)}$ has a smaller impact on $D^{2} / D_{0}^{2}$ and $X_{v}^{(s)}$. Also, the gasoline drop evolution seems to be more insensitive to $\theta_{v}^{(\infty)}$ and $\sigma_{v}^{(\infty)}$ (runs 11, 16, and 17) than the evolution of the diesel fuel drop. Finally, contrasting with the diesel fuel results, the initial stages of the gasoline drop evolution are very insensitive to the value of Lewis number, as shown in comparing results from runs 11,18 , and 19. This means that the exact knowledge of $\mathcal{D}_{\text {eff }}$ is relatively unimportant for initial $T_{d}, D^{2} / D_{0}^{2}$, or $\theta_{l}$ variation. However, $\theta_{v}^{(s)}$ and $\sigma_{v}^{(s)}$ display some sensitivity to Lewis number past the initial quarter of the drop lifetime.

\section{Simplified Models}

A comparison is shown in Fig. 11 of the four-pseudocomponent models and models M1 and M2 predictions with those of the discrete model. The four-pseudocomponentmodel is by far the worst in that it cannot even predict $T_{d}, D^{2} / D_{0}^{2}$ (Fig. 11a), or $X_{v}^{(s)}$ (Fig. 11b), which were shown to be calculated equally well by the singleand double- $\Gamma$-PDF. Moreover, the four-pseudocomponent model displays an unphysical nonmonotonic behavior in all quantities, and this makes it qualitatively inadequate to represent the discrete model evolution. Among the remaining two models, M2, which emphasizes a wider range of the light components, fares better than M1. Although not very adept in reproducing $\theta_{l}$ and $\sigma_{l}$ from the discrete model (Figs. 11c and 11d), it can predict $\theta_{v}^{(s)}$ and $\sigma_{v}^{(s)}$ reasonably well (Figs. 11e and 11f), which are the crucial information to describe the gas-phase composition. However, we note that even this model is incapable of describing the surface vapor discrete model PDF, as seen in Figs. $11 \mathrm{~g}$ and $11 \mathrm{~h}$, particularly, the relative magnitude of its peaks, which we showed embodies the important physics. Moreover, the choice of the two pseudocomponents is arbitrary, and it is uncertain how it influences the results. 


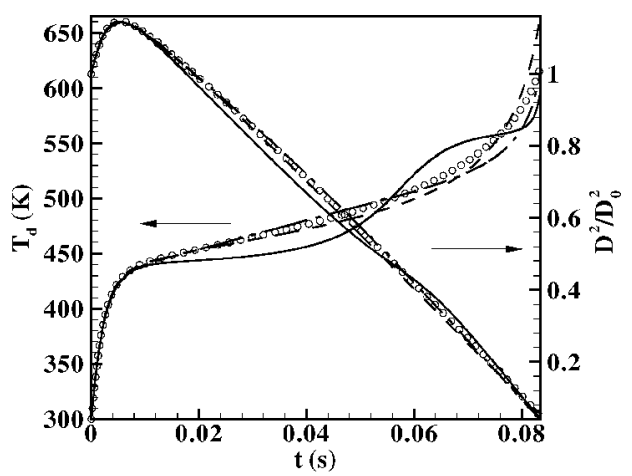

a)

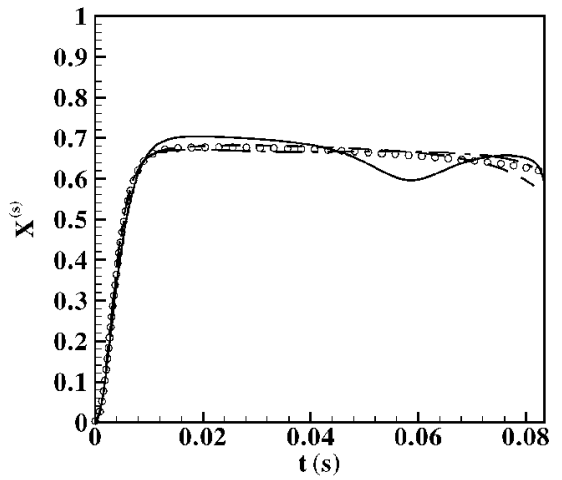

b)
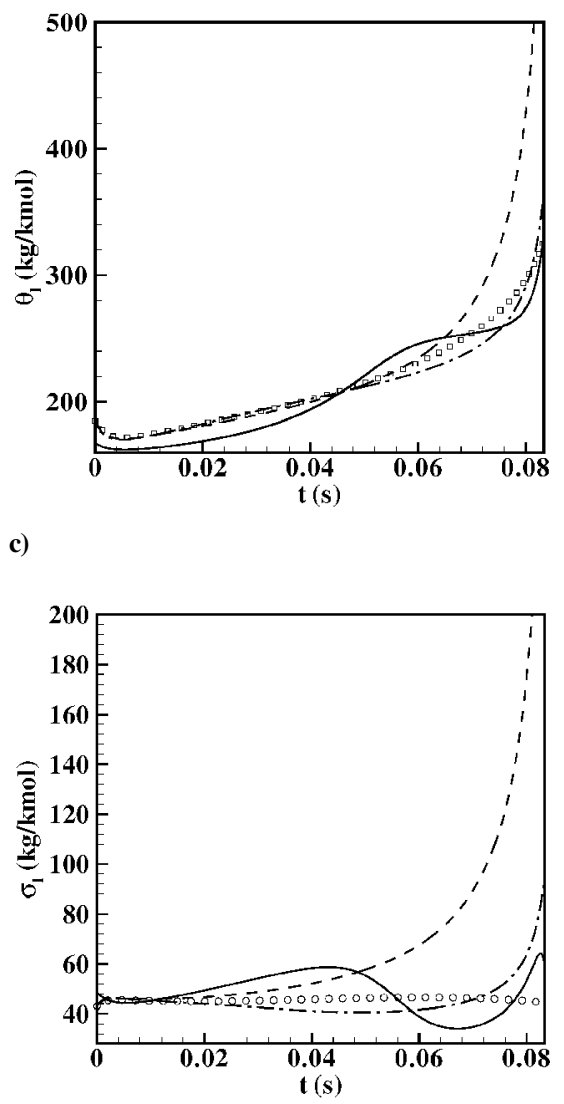

d)

Fig. 11 Diesel drop (run 2) timewise evolution according to the discrete model and three simplified models: a) drop temperature and relative surface area, b) drop surface vapor mole fraction, c) liquid mean molar weight, d) liquid PDF standard deviation, e) surface composition mean molar weight, f) surface composition PDF standard deviation, g) surface vapor PDFs at $60 \%$ residual drop mass, and h) surface vapor PDFs at $20 \%$ residual drop mass: ${ }^{\circ}$, discrete model; ——, pseudocomponent model; ---, M1 model; and - - -, M2 model.

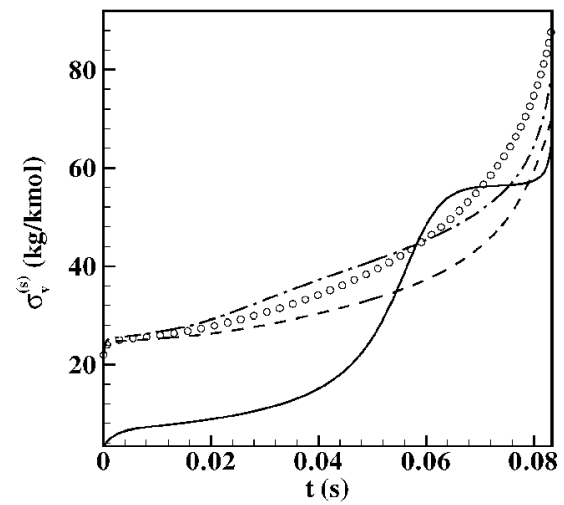

f)

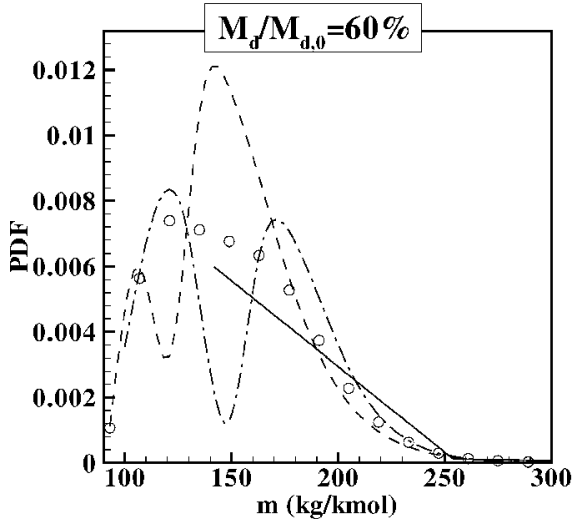

g)

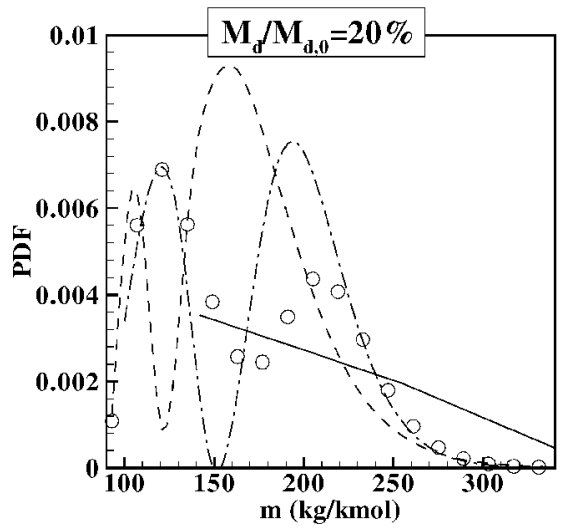

h)

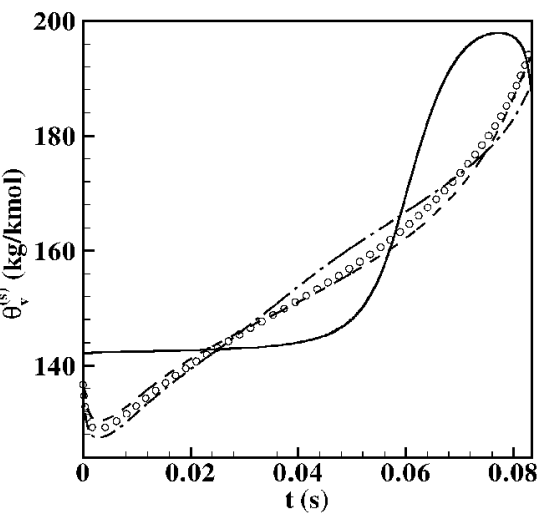

e) 


\section{Conclusions}

A model has been developed to describe the evaporation of a drop of an MC fuel containing a multitude of species. The model adopts a statistical representation through a distribution function, based on CT concepts. Unlike a precedent model, the present model is not based on the distribution function retaining its original single$\Gamma$-PDF form during drop evaporation. This new representation responds to the observation that, when the single- $\Gamma-\mathrm{PDF}$ is assumed to represent the fuel distribution during evaporation, it leads to strong departures from a discrete model results accounting for all individual species in the mixture. The discrete model was exercised for 32 paraffin species, making it numerically tractable, allowing the accounting of species of interest in petroleum fuels, and enabling the extraction of statistics that serve as the baseline results against which all other models are compared. These baseline results are necessary because it is also shown that quantities usually measured to describe drop evaporation, such as residual drop surface area, drop temperature, or vapor mass fraction at the drop surface, are insensitive to the drop and surrounding gas composition.

The new model describing drop evaporation is based on the evolution of an initial-liquid-fuel-composition single- $\Gamma$-PDF to a combination of two $\Gamma$-PDFs, a form called the double- $\Gamma$-PDF. This form is suggested by results from the discrete model showing that, when a drop is immersed into gaseous surroundings whose composition includes light species (as would be the case in sprays used in energy producing devices, because of the light species already evaporated from other drops), condensation of these light species creates a minor peak of the distribution at the lower end of the molar weight. To replicate this behavior, one must determine the double- $\Gamma$-PDF by computing its five parameters: two for each of the two $\Gamma$-PDFs and the height of the minor peak. This problem of determining the five parameters is reduced to an inverse mapping of the distribution first five moments to the five parameters of the double- $\Gamma$-PDF. This inverse mapping is accomplished only approximately; this is, however, not considered crucial to the final results because the discrete model PDF may actually not be entirely in double- $\Gamma$-PDF form.

Extensive comparisons of the double- $\Gamma$-PDF with the statistics from the discrete representation for diesel and gasoline fuels, representing different degrees of fuel volatility, show that the predictions of the model can be qualified as excellent for the composition of the vapor fuel at the drop surface that determines the vapor composition. Excellent predictions are also obtained for the mean molar weight of the liquid; however, the predictions of the liquid molar weight standard deviation is excellent to fair, depending on the situation. It is also shown that the accuracy of the predictions depends to a great extent on the double- $\Gamma$-PDF being able to capture the liquiddistributionminor peak that evolves as a result of condensation. This peak is minor for the liquid composition; however, for the vapor it may be of similar magnitude as the high-molar-weight peak during the early drop lifetime and even dominate the drop-surface vapor composition in the later stage of the drop lifetime.

When computational times of the models are compared, the discrete-species model is 10 times more computationally intensive than either of the PDF models, whereas the double- $\Gamma$-PDF is only about $5 \%$ more computationally intensive than the single- $\Gamma$-PDF. When it is considered that turbulent flow simulations with millions of single-component drops takes several thousand hours of CPU time, the CPU savings in adopting the PDF representation may enable computations that would otherwise be unfeasible.

Several approximate models of the double- $\Gamma$-PDF based on four rather than five parameters are also tested against the discrete models, and it is shown that they are unable to capture the crucial physics of drop evaporation.

\section{Appendix A: Correlations of Thermophysical Properties}

In the calculations, air is treated as a single pseudocomponent, inert carrier gas with an effective $T_{c}=133 \mathrm{~K}, m_{a}=29 \mathrm{~g} / \mathrm{mol}$, and ratio of heat capacities $\gamma_{a}=1.4$. Then, $\lambda_{a}=1.36 \times 10^{-2}(T /$ $\left.T_{c}\right)^{0.75} \mathrm{~W} / \mathrm{mK}$ and $C_{p}=\gamma_{a} R_{u} /\left(\gamma_{a}-1\right) m_{a} \mathrm{~J} / \mathrm{gK}$, where $R_{u}=$ $8.3142 \mathrm{~J} / \mathrm{mol} \mathrm{K}$.
We used information from the American Petroleum Institute ${ }^{33}$ to obtain the correlation $T_{b}\left(m_{i}\right)=241.4+1.45 m_{i} \mathrm{~K}$ and to develop of the following correlation $\lambda_{v}\left(m_{i}\right)=3.45 \times 10^{-3} m_{i}^{0.37}(T /$ $\left.T_{b}\right)^{n} \mathrm{~W} / \mathrm{mK}$, where $n=2.225 m_{i} /\left(m_{i}+19.245\right)$. The correlations for the heat capacities were those of Tamim and Hallett, ${ }^{18}$ namely $C_{p}\left(m_{i}\right)=\left(A_{p}+B_{p} m_{i}\right) R_{u} / m_{i} \mathrm{~J} / \mathrm{gK}$, where $A_{p}=$ $2.465-0.1144 T+1.759 \times 10^{-5} T^{2}-5.972 \times 10^{-9} T^{3}$, and $B_{p}=$ $-0.03561+9.367 \times 10^{-4} T-6.030 \times 10^{-7} T^{2}+1.324 \times 10^{-10} T^{3}$. For both $\lambda_{v}\left(m_{i}\right)$ and $C_{p}\left(m_{i}\right)$, the calculation is made at the surface conditions, that is, $T_{d}$ and $m_{v}^{(s)}$. Also following Ref. 18, $C_{l}=2.26-$ $2.94 \times 10^{-3} T_{d}+9.46 \times 10^{-6} T_{d}^{2} \mathrm{~J} / \mathrm{gK}$. To calculate properties for a mixture, we used mixing rules based on mass fractions.

\section{Appendix B: Relationships for a Double-Г-PDF}

The moments corresponding to the PDF given by Eq. (12) are

$$
\begin{aligned}
\xi_{n l}= & (1-\epsilon) \sum_{m=0}^{n} \frac{n !}{(n-m) ! m !} \beta_{1}^{n-m} \gamma^{m} \frac{\Gamma\left(\alpha_{1}+n-m\right)}{\Gamma\left(\alpha_{1}\right)} \\
& +\epsilon \sum_{m=0}^{n} \frac{n !}{(n-m) ! m !} \beta_{2}^{n-m} \gamma^{m} \frac{\Gamma\left(\alpha_{2}+n-m\right)}{\Gamma\left(\alpha_{2}\right)}
\end{aligned}
$$

presented consistent with Eq. (16). The first five moments that are here of interest are for $j \in[1,2], \xi_{1 l}^{(j)}=\theta_{j}, \xi_{2 l}^{(j)}=\theta_{j}^{2}+\sigma_{j}^{2}, \xi_{3 l}^{(j)}=$ $\theta_{j}^{3}+\sigma_{j}^{2}\left(3 \theta_{j}+2 \beta_{j}\right), \quad \xi_{4 l}^{(j)}=\theta_{j}^{4}+3 \sigma_{j}^{4}+2 \sigma_{j}^{2}\left(3 \theta_{j}^{2}+4 \theta_{j} \beta_{j}+3 \beta_{j}^{2}\right)$, and $\xi_{5 l}^{(j)}=\theta_{j}^{5}+5 \sigma_{j}^{4}\left(3 \theta_{j}+4 \beta_{j}\right)+2 \sigma_{j}^{2}\left(5 \theta_{j}^{3}+10 \theta_{j}^{2} \beta_{j}+15 \theta_{j} \beta_{j}^{2}+\right.$ $\left.12 \beta_{j}^{3}\right)$. From the definition of Eq. (17),

$$
\begin{aligned}
& -\xi_{3}^{\prime} /(\theta-\gamma)=\beta^{\prime}\left[\beta+\Delta^{\prime}-\left(\theta-\gamma-\Delta^{\prime}\right)\left(3 \omega+2 \omega^{2}\right)\right] \\
& -\xi_{4}^{\prime} /(\theta-\gamma)=\beta^{\prime}\left\{\beta(7 \beta+4 \theta)+2 \beta^{\prime}(\theta-\gamma-\beta)\right. \\
& +\Delta^{\prime}\left(6 \beta+4 \theta-4 \beta^{\prime}\right)-\left(\Delta^{\prime}\right)^{2}-\left(\theta-\gamma-\Delta^{\prime}\right) \\
& \quad \times\left[\omega\left(22 \beta+12 \theta-16 \beta^{\prime}-6 \Delta^{\prime}\right)+\omega^{2}\left(18\left(\beta-\beta^{\prime}\right)\right.\right. \\
& \left.\left.\left.+11\left(\theta-\Delta^{\prime}\right)-3 \gamma\right)-6 \omega^{3}\left(\beta^{\prime}+\Delta^{\prime}\right)\right]\right\}
\end{aligned}
$$

where $\Delta^{\prime} \equiv \beta^{\prime}+\theta^{\prime}$ and $\Delta^{\prime} \geq 0$ for $\epsilon \leq 0.5$. To determine the inverse mapping, a cubic algebraic equation $A v^{3}+B v^{2}+C v-\Lambda=0$ is solved, where $\Lambda$ was defined in Sec. II.C and $A(\omega)=(1+3 \omega) K_{1}^{2}$ and $\quad B(\omega)=2 K_{1}^{2} K_{2} /(\beta+\theta-\gamma)-K_{1}\left(1+3 \omega+K_{3}+K_{1} \Lambda\right)$, $C(\omega)=K_{3}+2 K_{1} \Lambda+2 \omega(1+\omega)(2+3 \omega) K_{2} /(\beta+\theta-\gamma)$, with $K_{1} \equiv(1+\omega)(1+2 \omega), K_{2} \equiv-\xi_{3}^{\prime} /[(\theta-\gamma)(\beta+\theta-\gamma)]$, and $K_{3} \equiv$ $K_{1}+2 \omega^{2}(2+3 \omega)-2 \omega(1+\omega)(2+3 \omega) \beta /(\beta+\theta-\gamma)$. The solution for $v$ determines unknowns $\beta^{\prime}=K_{2} /\left(1-K_{1} v\right)$ and $\Delta^{\prime}=\theta-$ $\gamma-(\beta+\theta-\gamma) v$.

\section{Acknowledgments}

This research was performed at the Jet Propulsion Laboratory of the California Institute of Technology, under the partial sponsorship of U.S. Department of Energy (DOE), with Neil Rossmeissl and Roxanne Danz (DOE Headquarters) and D. Hooker (DOE Golden Center) serving as Contract Monitors, under an agreement with NASA. Additional sponsorship was provided by the Donors of The Petroleum Research Fund administered by the American Chemical Society through a grant to J. Bellan that supported a California Institute of Technology Postdoctoral Fellow (P. C. Le Clercq).

\section{References}

${ }^{1}$ Hanson, S. P., Beer, J. M., and Sarofim, A. F., "Non-Equilibrium Effects in the Vaporization of Multicomponent Fuel Droplets," Proceedings of the Combustion Institute, Vol. 19, 1982, pp. 1029-1036.

${ }^{2}$ Sorbo, N. W., Law, C. K., Chang, P. Y., and Steeper, R. R., "An Experimental Investigation of the Incineration and Incinerability of Chlorinated Alkane Drops," Proceedings of the Combustion Institute, Vol. 22, 1989, pp. 2019-2026.

${ }^{3}$ Randolph, A. L., Makino, A., and Law, C. K., "Liquid-Phase Diffusional Resistance in Multicomponent Droplet Gasification," Proceedings of the Combustion Institute, Vol. 21, 1986, pp. 601-608.

${ }^{4}$ Wang, C. H., Liu, X. Q., and Law, C. K., "Combustion and Microexplosion of Freely Falling Multicomponent Droplets," Combustion and Flame, Vol. 56, No. 2, pp. 175-197. 
${ }^{5}$ Yang, J. C., and Avedesian, C. T., "The Combustion of Unsupported Heptane/Hexadecane Mixture Droplets at Low Gravity," Proceedings of the Combustion Institute, Vol. 22, 1988, pp. 2037-2044.

${ }^{6}$ Jackson, G. S., Avedesian, C. T., and Yang, J. C., "Observations of Soot During Droplet Combustion at Low Gravity-Heptane and Heptane Monochloroalkane Mixtures," International Journal of Heat and Mass Transfer, Vol. 35, No. 8, 1992, pp. 2017-2033.

${ }^{7}$ Gökalp, I., Chauveau, C., Berrekam, H., and Ramos-Arroyo, N. A., "Vaporization of Miscible Binary Fuel Droplets Under Laminar and Turbulent Convective Conditions," Atomization and Sprays, Vol. 4, 1994, pp. 661-676.

${ }^{8}$ Marchese, A. J., Dryer, F. L., Colantonio, R. O., and Nayagam, V., "Microgravity Combustion of Methanol and Metahnol/Water Droplets: Drop Tower Experiments and Model Predictions," Proceedings of the Combustion Institute, Vol. 26, 1996, pp. 1209-1217.

${ }^{9}$ Wood, B. J., Wise, H., and Inami, S. H., "Heterogeneous Combustion of Multicomponent Fuels," Combustion and Flame, Vol. 4, No. 3, 1960, pp. $235-242$.

${ }^{10}$ Wong. S. C., and Lin, A. C., "Internal Temperature Distributions of Droplets Vaporizing in High-Temperature Convective Flows," Journal of Fluid Mechanics, Vol. 237, 1992, pp. 671-687.

${ }^{11}$ Law, C. K., Prakash, S., and Sirignano, W. A., "Theory of Convective, Transient, Multicomponent Droplet Vaporization," Proceedings of the Combustion Institute, Vol. 16, 1976, pp. 605-617.

${ }^{12}$ Lara-Urbaneja, P., and Sirignano, W. A., "Theory of Transient Multicomponent Droplet Vaporization in a Convective Field,' Proceedings of the Combustion Institute, Vol. 18, 1981, pp. 1365-1374.

${ }^{13}$ Tong, A. Y., and Sirignano, W. A., "Multicomponent Droplet Vaporization in a High Temperature Gas," Combustion and Flame, Vol. 66, 1986, pp. 221-235.

${ }^{14}$ Tong, A. Y., and Sirignano, W. A., "Multicomponent Transient Droplet Vaporization with Internal Circulation: Integral Equation Formulation and Approximate Solution," Numerical Heat Transfer, Vol. 10, 1986, pp. 253-278.

${ }^{15}$ Megaridis, C. M., and Sirignano, W. A., "Numerical Modeling of a Vaporizing MulticomponentDroplet," Proceedings of the Combustion Institute, Vol. 23, 1990, pp. 1413-1421.

${ }^{16}$ Wood, C. P., McDonell, V. C., Smith, R. A., and Samuelsen, G. S., "Development and Application of a Surrogate Distillate Fuel," Journal of Propulsion, Vol. 5, No. 4, 1989, pp. 399-405.

${ }^{17}$ Schultz, W. D., "Oxidation Products of a Surrogate JP-8 Fuel," Symposium on Structure of Jet Fuels III, Div. of Petroleum Chemistry, Inc., American Chemical Society, 1991.

${ }^{18}$ Tamim, J., and Hallett, W. L. H., "A Continuous Thermodynamics Model for Multicomponent Droplet Vaporization," Chemical Engineering Science, Vol. 50, No. 18, 1995, pp. 2933-2942.
${ }^{19}$ Hallett, W. L. H., "A Simple Model for the Vaporization of Droplets with Large Numbers of Components," Combustion and Flame, Vol. 121, 2000, pp. 334-344.

${ }^{20}$ Lippert, A. M., "Modeling of Multicomponent Fuels with Application to Sprays and Simulation of Diesel Engine Cold Start," Ph.D. Dissertation, Univ. of Wisconsin, Madison, WI, 1999.

${ }^{21}$ Lippert, A. M., and Reitz, R. D., "Modeling of Multicomponent Fuels Using Continuous Distributions with Application to Droplet Evaporation and Sprays," Society of Automotive Engineers, SAE Paper 972882, 1997.

${ }^{22}$ Bowman, J. R., and Edmister, W. C., "Flash Distillation of an Indefinite Number of Components," Industrial and Engineering Chemistry, Vol. 43 , 1951, pp. 2625-2628.

${ }^{23}$ Edmister, W. C., and Bowman, J. R., "Equilibrium Conditions of Flash Vaporization of Petroleum Fractions," Chemical Engineering Progress Symposium Series, Vol. 48, American Inst. of Chemical Engineers, New York, 1952, pp. 46-51.

${ }^{24}$ Aris, R., and Gavalas, G. R., "On the Theory of Reactions in Continuous Mixtures," PhilosophicalTransactions of the Royal Society of London, Series A: Mathematical and Physical Sciences, Vol. A 260, 1966, pp. 351-393.

${ }^{25}$ Briano, J. G., and Glandt, E. D., "Molecular Thermodynamics of Continuous Mixtures," Fluid Phase Equilibria, Vol. 14, 1983, pp. 91-102.

${ }^{26}$ Cotterman, R. L., Bender, R., and Prausnitz, J. M., "Phase Equilibria for Mixtures Containing Very Many Components. Development and Application of Continuous Thermodynamics for Chemical Process Design," Industrial and Engineering Chemistry Process Design and Development Vol. 24, American Chemical Society, Washington, DC, 1985, pp. 194-203

${ }^{27}$ Gal-Or, B., Cullinan, H. T., Jr., and Galli, R., "New ThermodynamicTransport Theory for Systems with Continuous Component Density Distributions," Chemical Engineering Science, Vol. 30, 1975, pp. 1085-1092.

${ }^{28}$ Rätzsch, M. T., and Kehlen, H., "Continuous Thermodynamics Model of Complex Mixtures,” Fluid Phase Equilibria, Vol. 14, 1983, pp. 225-234.

${ }^{29}$ Whitson, C. H., "Characterizing Hydrocarbon Plus Fractions," Society of Petroleum Engineers Journal (Dallas), Vol. 23, 1983, pp. 683-694.

${ }^{30}$ Chou, G. F., and Prausnitz, J. M., "Adiabatic Flash Calculations for Continuous or Semicontinuous Mixtures Using an Equation of State," Fluid Phase Equilibria, Vol. 30, 1986, pp. 75-82.

${ }^{31}$ Williams, F. A., Combustion Theory, Addison-Wesley, Reading, MA, 1965 , p. 47

${ }^{32}$ Law, C. K., "Quasi-Steady Droplet Vaporization Theory with Property Variations," Physics of Fluids, Vol. 11, No. 18, 1975, pp. 1426-1432.

${ }^{33}$ Technical Data Book-Petroleum Refining, 5th ed., American Petroleum Inst., Washington, DC, 1992, pp. 12-3, 12-21.

P. Givi Associate Editor 